IOS Press

\title{
Hypothesis
}

\section{Shear-Induced Amyloid Formation in the Brain: III. The Roles of Shear Energy and Seeding in a Proposed Shear Model}

\author{
Conrad N. Trumbore* \\ Department of Chemistry and Biochemistry, University of Delaware, Newark, DE, USA
}

Accepted 20 June 2018

\begin{abstract}
If cerebrospinal and interstitial fluids move through very narrow brain flow channels, these restrictive surroundings generate varying levels of fluid shear and different shear rates, and dissolved amyloid monomers absorb different shear energies. It is proposed that dissolved amyloid- $\beta$ protein $(A \beta)$ and other amyloid monomers undergo shear-induced conformational changes that ultimately lead to amyloid monomer aggregation even at very low brain flow and shear rates. Soluble $\mathrm{A} \beta$ oligomers taken from diseased brains initiate in vivo amyloid formation in non-diseased brains. The brain environment is apparently responsible for this result. A mechanism involving extensional shear is proposed for the formation of a seed $A \beta$ monomer molecule that ultimately promotes templated conformational change of other $A \beta$ molecules. Under non-quiescent, non-equilibrium conditions, gentle extensional shear within the brain parenchyma, and perhaps even during laboratory preparation of $A \beta$ samples, may be sufficient to cause subtle conformational changes in these monomers. These result from brain processes that significantly lower the high activation energy predicted for the quiescent $A \beta$ dimerization process. It is further suggested that changes in brain location and changes brought about by aging expose $\mathrm{A} \beta$ molecules to different shear rates, total shear, and types of shear, resulting in different conformational changes in these molecules. The consequences of such changes caused by variable shear energy are proposed to underlie formation of amyloid strains causing different amyloid diseases. Amyloid researchers are urged to undertake studies with amyloids, anti-amyloid drugs, and antibodies while all of these are under shear conditions similar to those in the brain.
\end{abstract}

Keywords: Amyloid- $\beta$, cerebrospinal fluid, glymphatic pathway, interstitial fluid, shear energy, templated conformation change

\section{INTRODUCTION}

Despite more than a century of research into the causes of devastating neurodegenerative amyloid disorders such as Alzheimer's disease (AD) and Parkinson's disease, “... no disease-modifying drug has yet entered clinical use. This situation can be attributed, at least in part, to an incomplete

\footnotetext{
*Correspondence to: Conrad N. Trumbore, 441 Crosslands Drive, Kennett Square, PA 19348, USA. Tel.: +1 610388 7093; Fax: +1 610388 5691; E-mail: conradt@udel.edu.
}

understanding of the fundamental origins of such diseases" [1]. There have been many technical advances in the biological, biochemical, biophysical, theoretical, and medical fields that have been utilized to comprehend the etiology of these diseases [2]. There has been a significant shift from an earlier emphasis on amyloid fibrils produced by an amyloid chemical cascade sequence [3] to an emphasis on neurotoxic amyloid- $\beta$ protein $(A \beta)$ oligomers [4]. Why, then, is there so much difficulty in the search for drugs against these toxic oligomers? Could there be a mechanism that is being overlooked? 
This paper offers insights into the potentially important role that brain liquid shear may play as a source of mechanical energy that is proposed to be important in the etiology of amyloid diseases. The crowded brain environment [5-7] may play a critical role through mechanical molecular distortion in creating shear-activated oligomers that are able to undergo the process of pathological protein templating [8], a seeding process that mimics the conformation altering-properties of prions [9]. The suggestion is made here that seeding experiments using oligomers obtained from a diseased animal to induce amyloid aggregation in an animal with no disease are a possible key to understanding the potential relationship between shear and amyloid diseases [9]. That key is proposed to be the highly restricted flow of interstitial fluid (ISF) within the brain, producing different types of liquid shear that generate mechanical energy that distorts amyloid monomers and resulting in aggregated oligomers.

While accepting the possibility of both quiescent and non-quiescent solution formation of in vivo oligomer seeds, this paper proposes that certain types of laboratory shear experiments may be able to produce short-lived monomer and dimer seeds. These seeds then create oligomers that mimic those oligomers extracted from amyloid diseased brains. It is suggested that following the in vivo enzymaticinduced liberation of $A \beta$ from amyloid- $\beta$ protein precursor, some of these newly formed $A \beta$ molecules are quickly subject to both shear forces and membrane surfaces. Here they are adsorbed temporarily and quickly lose their shear energy-unless they encounter another sheared $A \beta$ molecule. The result of such bimolecular $A \beta$ encounters on the membrane may be seed dimers. These ultimately form seed oligomers through the conformational conversion of other encountered $A \beta$ molecules or a simultaneous conformational change of two excited $A \beta$ monomer molecules (D. Teplow, private communication). Furthermore, the proposal is made that the amount and type of shear energy generated in different brain regions can form shear-excited monomers with different conformations that create different oligomers strains, resulting in different types of amyloid disease.

\section{Seeding with conformation-altered, brain-derived amyloids}

It has been proposed that $A \beta$ aggregate seeds are probably capable of exhibiting prion-like behavior
$[9,10]$. That is, if the in vivo amyloid monomer or oligomer has undergone a specific type of conformational transformation that results in an oligomer seed [4], that oligomer has the capability to act as a template or seed in its reactions with other non-transformed $\mathrm{A} \beta$ monomers and transform their conformations into the same conformation type as the seed. In essence, there is a "conformation infection" of native $A \beta$ molecules, with spreading of this same "misfolded" conformational change throughout the body resulting in toxic consequences.

Another designation for this seeding behavior is "corruptive protein templating" [9, 10]. Regardless of the terminology, if soluble low molecular weight seeds isolated from the brain of one diseased experimental animal are injected into another disease-free transgenic animal, these "abnormally folded" proteins induce the "misfolding" and subsequent aggregation of like conformationally-transformed proteins leading to the formation of characteristic plaque lesions. These transformed conformations have a characteristic cross- $\beta$ structure that is the same as that of the seed and behave in much the same manner as in the strain-specific infectivity observed with prion proteins $[11,12]$.

What is most striking about the above seeding results is that synthetic and recombinant protein aggregates are not nearly as effective in seeding as those derived from the brain $[9,10]$. It is found that the most effective brain-derived seeds are those of low molecular weight. However, a conformationtransformed monomer has not yet been isolated or detected.

Thus, the question arises, what is responsible in the brain for the initial monomer, dimer, or oligomer conformation change that causes the formation of a templating oligomer seed? Is this original templating confined to the brain? Can it be reproduced in the laboratory in some other manner? This paper suggests that it may well be possible using liquid shear in highly confined spaces mimicking those found within the very narrow flow channels of the brain.

\section{IN VITRO RESEARCH CHALLENGES}

\section{Toxic oligomers}

One reason for the slow pace of basic amyloid research is the large number of unstable oligomers discovered with differing degrees of toxicity formed under different experimental [2] and cellular conditions $[13,14]$. Reactions producing oligomers are 
reported to be reversible, making it difficult to sort out mechanisms for the stepwise formation of oligomers, starting with the amyloid monomer.

Experimental tools such as nuclear magnetic resonance, Fourier transform infrared, circular dichroism, and light scattering have been used to study amyloid oligomer intermediates [2] but no detailed chemical mechanisms have been suggested. This is because there are apparently no easily-studied, step-by-step conformational changes from random coil to cross- $\beta$ states between the native monomer and a low molecular weight oligomer. It has been difficult to utilize theoretical molecular calculations [14-16] to predict early stochastic thermal conformational changes that convert intrinsically disordered protein (IDP) amyloid monomers into the cross- $\beta$ conformations found in amyloid oligomers, protofibrils, and fibrils.

There are currently two different mechanisms proposed regarding the origin of in vitro $\mathrm{A} \beta$ oligomers $[2,4,17]$. One is designated nucleated polymerization and the other nucleated conformational conversion. The former theory suggests that at low $A \beta$ concentration, polymerization of $A \beta$ monomers is initiated in a rate-determining step with the formation of a nucleus that gives rise to fibrils through successive monomer additions in an $A \beta$ linear polymer elongation process. Relatively low concentrations of oligomers are predicted in this pathway. In the other theory, favored at high $A \beta$ concentration, $A \beta$ aggregates act as intermediates on the path to fibril formation and are said to undergo a rate-determining conformational change to a cross beta conformation in order to ultimately obtain the oligomer and final fibril product. Both of these theories have a dual dependence on temperature and $\mathrm{A} \beta$ concentration in equilibria involving monomers and oligomers. There is limited experimental information regarding specific chemical reactions in either of these mechanisms.

\section{Initiation of oligomer formation and the amyloid cascade}

There is little clarity about rate-determining chemical or biological events that initiate in vivo amyloid reactions leading to the formation of neurotoxic oligomers, especially those resulting in the early neuron damage that precedes the devastating cognitive effects of AD. A recent review suggested that “... the most effective procedures for the prevention and treatment of misfolding diseases are likely to be those that address the earliest events in their development..." [18]. Thus in this paper, a chief concern is finding the answer to the question, what are these "earliest events"?"

Under quiescent laboratory conditions, it would appear that there is a slow, rate-determining conformational change either of a monomer or an aggregated group of monomers from random coil with some alpha-helical structure, to a cross- $\beta$ conformation [19]. Theoretical and experimental estimates of the activation enthalpy and free energy for the elongation pathway for the addition of monomer to a growing amyloid are comparatively high [20]. It has been proposed that highly complex aggregation processes such as primary and secondary nucleation, and elongation take place during the lag time preceding the aggregation process [21].

\section{Sensitivity of the $A \beta_{1-42}$ isoform}

Of the two natural $A \beta$ isoforms, the $A \beta_{42}$ isoform is more difficult to work with experimentally because $A \beta_{42}$ is more sensitive to effects induced by agitation and shear than is $A \beta_{40}$ [22]. However, the $A \beta_{42}$ isoform is the dominant one found in senile plaque, whereas the other major isoform, $A \beta_{40}$, is the dominant isoform in vascular deposits. In the first paper in this series, the author has proposed a mechanism explaining this experimental fact based on $A \beta$ isoform differences in shear sensitivity in different parts of the brain [23]. This proposed mechanism depends on differences in flow rates of cerebrospinal fluid (CSF) and the slower ISF flow in the brain parenchyma. Fluid shear critically depends on the ISF flow rate and geometry of ISF flow channels in different parts of the brain. However, brain parenchymal ISF flow rates have not yet been reported, although the very small dimensions of possible ISF flow paths have been predicted [24], and research is ongoing on the measurement of CSF rates around arteries entering the rat brain (D. Kelly and J. Thomas, private communication). Shear studies at very low shear rates have demonstrated both shear-induced protein conformational changes [25] and shear-induced $A \beta$ cascade reactions [22].

\section{Misfolding as the cause of amyloid diseases}

It is repeatedly suggested in explaining the origins of amyloid diseases that the ultimate cause is a "misfolded" amyloid monomer or oligomer that initiates the amyloid cascade leading to toxic oligomers, protofibrils, fibrils, and ultimately to amyloid plaque. In laboratory quiescent solution studies, there appears 
to be an inferred random thermal stochastic molecular collision event involving two or more very high energy molecules that leads to a critical "misfolding" event. Alternatively, in vivo it has been assumed that there is a flaw in an in vivo folding or refolding process and that the origin of this misfolded protein is due to a malfunctioning brought on by aging that fails to recognize and eliminate the misfolded protein [26]. That is, there is assumed to be either a malfunction in the original protein folding process or in the refolding of an $A \beta$ molecule that brings about the formation of toxic amyloid oligomers.

Nearly all of the reported amyloid laboratory experiments have been performed in quiescent solutions. This paper questions whether experiments with quiescent solutions should be the exclusive brain model for the types of fluid processes that may be taking place in the crowded conditions within the brain parenchyma. It suggests that, in addition, aggregation processes should also be included that incorporate a flow condition that includes fluid shear. It is proposed that shear is present in all ISF solutions containing amyloid monomers [23]. This shear arises from the highly restricted flow through the many flow-impeding objects present within the brain parenchyma. It is further proposed that such brain liquid shear is, at least in part, the cause of the "misfolding" of amyloid monomers.

\section{IN VIVO SHEAR}

\section{The crowded brain environment}

The extracellular space (ECS) in which ISF flows is very limited and very crowded with dense packing of neurons with their abundant neurites, as well as glial projections from astrocytes, microglia, and other flow-blocking molecular assemblies. The ECS flow paths are not only narrow and constricted, but are tortuous, with many detours around projections from neuron and other glial surfaces as well as protein complexes projecting from and embedded in membranes and proteins suspended within the ECS [24]. Dobson [27] claims that within the crowded brain environment, the amyloid state of a protein, except in rare cases where this protein has a functional role, is intrinsically misfolded and inherently prone to interact "inappropriately" within the crowded parenchymal environment in a toxic manner. In such an environment, it is possible that the amyloid monomer is thermodynamically unstable [5]. Thus, sheared ISF solutions may be more prone to formation of excited free energy states than in a less complex aqueous solution. Studies of amyloid fibrils are found to be strongly influenced by crowding and demonstrate markedly different amyloid propagation under such conditions [7].

\section{The brain environment results in mild fluid shear}

$\mathrm{A} \beta$-containing CSF and ISF solutions have been reported to be flowing through highly restricted flow paths, with different glymphatic flow rates in different regions [28]. Although there is controversy over the nature, the speed, and flow oscillations, including possible reversals of the direction of this ISF and CSF flow [29, 30], there is a high probability that flowing $A \beta$-containing fluids generate at least some gentle shear within the brain parenchyma [23]. Such flow may also have an important circadian pattern in that CSF glymphatic flows providing brain $\mathrm{A} \beta$ clearance have been demonstrated to be much faster during rodent sleep than wakeful periods [31].

Mild shearing of $\mathrm{A} \beta$-containing solutions has been shown to be sufficient to initiate fibril formation despite the very low total amount of energy and rate of energy input, which is reported to be less than that needed for the breakage of one hydrogen bond per molecule [22]. There is a recent report that microfluid flow of $A \beta$, modeling blood flow through very narrow flow paths, induced $A \beta$ aggregation [32]. A critical paper reports that very gentle shear induces spectral changes that indicate subtle conformational changes in many different types of proteins [25], including $A \beta$ (L. Ashton, private communication). If shear-excited states have long relaxation times, the total time during which a molecule is exposed to even very low fluid shear rates is important and can cause protein aggregation. A recent paper demonstrates that a wide variety of non-IDP proteins undergo aggregation when exposed to long-term extensional shear [33] (See discussion below of different types of shear). Thus, there is ample experimental evidence that shear could be an important factor in amyloid aggregation within brain parenchyma, even at very low flow and shear rates.

Thus, in the opinion of this author, any realistic experimental in vitro model of brain amyloid processes needs to include at least some stringent fluid flow restrictions similar to those found in the brain. Furthermore, any studies of anti-amyloid or other drugs being tested for AD should also be exposed to the same flow-restricted, shear-producing environment. This includes anti-amyloid antibodies, whose 
conformations have also recently been reported to be shear-sensitive [33]. Although the effects would be much smaller, intracellular cytoplasmic, lysosome or endosome shear processes, arising from subtle conformation changes noted by Ashton et al., should not be neglected. Since ISF contains many different proteins, shear studies ultimately should explore the possibilities of mixed protein shear-induced aggregation products, e.g., $\mathrm{A} \beta$ and phosphorylated tau.

\section{Role of variable shear in $A \beta$ aggregation in the brain}

This current paper considers the possible consequences of variable energy levels of the liquidsheared $A \beta$ and other amyloid monomer proteins in the brain environment. The proposal will be made below that shear energy levels in different brain regions lead to different amyloid monomer molecule conformations, which lead to different strains that cause different medical outcomes. Justification for this proposal comes from the relatively recent realization that, under appropriate conditions, many different types of proteins can be converted into an amyloid state [27]. A key question is: What are the appropriate conditions that cause the conversion of polypeptide chains into a cross- $\beta$ conformation that appears to be the precondition for amyloid formation? One possible answer is presented below from the point of view of the various shear energy types and energy states involved within the brain.

\section{PROPOSED SHEAR MODEL}

\section{Protein folding and oligomer formation}

Structurally, all amyloid proteins have a common variable length backbone $(-\mathrm{N}-\mathrm{C}-\mathrm{C}-)_{\mathrm{n}}$, where $\mathrm{n}$ equals the number of joined amino acids, each containing one of 20 different $R$ groups attached to that backbone, whose unique side group sequence spread along the total length of the backbone determines the structures of a large variety and number of protein molecules. According to their unique sequence of $\mathrm{R}$ groups, these different linear structures fold into various unique, comparatively stable conformations designated as "native" proteins, as shown in Fig. 1. There is a very high activation energy for the conversion of two $A \beta$ molecules into a dimer molecule with a lower free energy per $A \beta$ molecule (Fig. 1).

However, as shown by the dashed line in Fig. 1, this high activation free energy is herewith proposed to be drastically reduced by mechanical liquid shearing of the dissolved $A \beta$ molecule, thereby lowering the height of an otherwise trapping high activation energy "dam." This barrier reduction, a shear-induced catalytic effect, is proposed as a critical driving force in the brain for inducing the aggregation of $\mathrm{A} \beta$ to form toxic oligomers. However, as is also depicted in Fig. 1, seeding amyloid monomer solutions with exogenous brain-derived oligomers also drastically lowers this barrier and promotes a catalytic, conformation-altering incorporation of these monomers in the formation of aggregation products.

It is proposed that the shear-induced brain dimer seed reproduces its unique conformation through corruptive protein templating in the brain leading to oligomers responsible for toxic biological processes. Furthermore, it is proposed that the mechanism of this initial conversion of the monomer to a dimer is a shear-induced conformational change that facilitates the formation of a cross beta oligomer structure.

Thus, initial shearing of monomer combined with shear product oligomer seeding can participate in downhill free energy chemical reactions in which subsequent monomers are conformationally converted and incorporated into an amyloid aggregate. The rate of this aggregation is dependent on seed and amyloid concentrations as well as temperature, $\mathrm{pH}$, ionic strength, etc. Later steps in this aggregation reaction may also be dependent on shear conditions. In this proposal, local seed concentration is dependent on the shear map of the brain. The critical shear properties depend on local shear rates and accumulated shear affecting a given molecule as a function of shear exposure time. Accumulated shear equals shear rate times the time of exposure to shear conditions.

\section{The initiating events in oligomer and fibril formation}

Thus, the combination of shear-based reduction in the activation energy barrier between monomer and dimer, allowing seed dimer and toxic oligomer seed formation, are proposed as the initiating events leading ultimately to amyloid disease. The rate-determining process is proposed to be the shearinduced lowering of the high activation energy barrier to allow fast formation of an altered-conformation amyloid monomer leading to formation of a cross beta conformation dimer and subsequent formation of a toxic oligomer seed. 


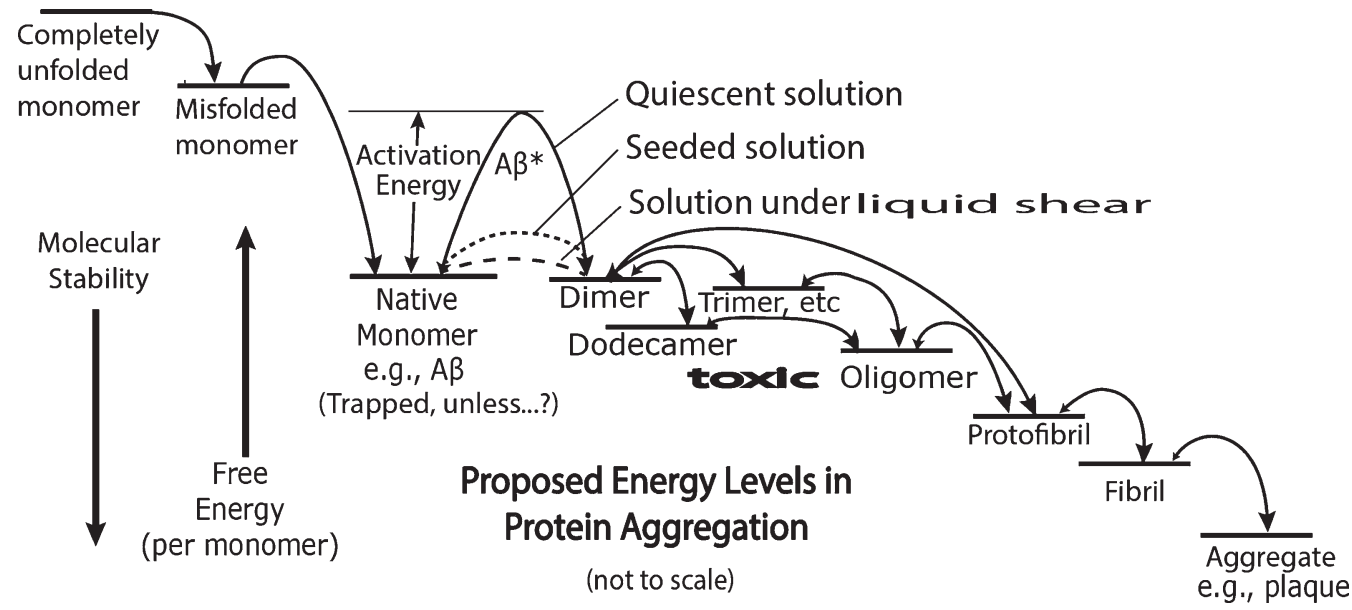

Fig. 1. Energy diagram representing a highly simplified, symbolic overview of two processes, protein folding and amyloid cascade aggregation proceeding from native monomer through the formation of toxic oligomers, fibrils, to aggregates. The free energy level scale is arbitrary and not to scale. The free energy and molecular stability arrows are arbitrary in size but not direction. As indicated with the vertical arrows on the left, the stability of the monomer increases with decreasing free energy. The heights of curved arrows above straight line energy levels represent speculative comparative activation energies. The relative sizes of the arrow heads in the double headed arrows indicate speculative relative rates in the equilibrium between each of the two chemical species. The dashed line between monomer and dimer represents the free energy of the monomer as it is transformed in a non-equilibrium shear energy process probably from a predominantly alpha to a predominantly cross beta conformation. The dotted line represents the lowered activation energy when exogenous oligomers are added to monomer solutions and catalyze further aggregation. The single line energy labels "Dimer", "Trimer, etc." do not reveal the complexity of different possible energy levels and conformations of in-register and out-of-register conformations. The "(Trapped. Unless ... )" label under the "Native Monomer" energy level implies there are means (e.g., shearing and seeding) by which the very high activation energy barrier can be circumvented and aggregation commences.

\section{Intrinsically disordered proteins}

In addition to other amyloids, $A \beta$ is a member of the group of proteins designated as IDP [34]. Unlike many other proteins, the structure of IDPs cannot be completely determined using X-ray diffraction because they do not have one distinct conformation. Rather, the IDP molecule samples a number of different conformations in a short period of time and might be described as a "floppy," rather flexible molecule with relatively short structured sections such as $\alpha$ helices and short beta sheet segments.

Since conformation determines biological function, proteins have many different conformations leading to many different functions. Many non-IDPs have only one native state conformation, designated "F" in the right side of Fig. 2 as a single ground state energy line and a free energy folding diagram with one major minimum [35], illustrated symbolically in Fig. 2 right and below. The squiggles in the right diagram represent highly unstable states in the free energy protein folding "funnel" diagram [35] However, the "floppy" protein IDP protein class such as $\mathrm{A} \beta$ are represented on the left side of Fig. 2 as multiple, relatively flat ground state energy levels $[36,37]$.

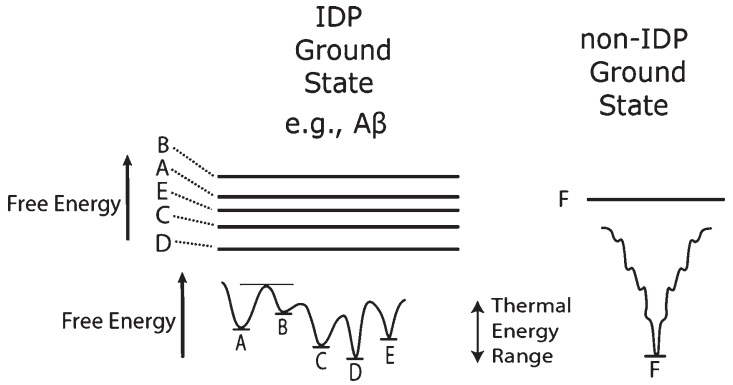

Fig. 2. Distinction between intrinsically disordered protein (IDP) and non-IDP ground free energy states, that is, lowest free energy states. IDPs such as $\mathrm{A} \beta$ are highly flexible molecules that have more than one ground state at normal temperatures. These molecules constantly change their conformations from state to state, e.g., A to $\mathrm{B}$ to $\mathrm{C}$, etc., as they receive and give up energy in collisions with their surroundings. They are not without a conformation and are not really "misfolded." They merely populate many different fairly loosely structured conformational states as a function of time. However, the relative depths of the accessible free energy states determine the lengths of time a single molecule spends within those states. Thus, an IDP molecule will spend more time in the D state than in the B state. In contrast, a non-IDP molecule with a single ground conformational state has only one stable conformation $(\mathrm{F})$ at normal temperatures. The double arrow "Thermal Energy Range" symbolizes the variable energy range of molecules undergoing Brownian motion. 
The IDP A $\beta$ has numerous metastable ground state conformations [14]. That is, because the ground states are very close together in free energy, these molecules are constantly changing their conformations from one of these metastable states to another, each with a slightly different energy minimum.

When a freshly synthesized linear protein is folded into its native conformation, significant amounts of energy are given off due to the protein chain folding because of multiple types of intramolecular attractive forces. Therefore, it must follow that, if one starts with a completely folded native monomer molecule, one must provide a significant amount of activation energy to break however many bonds are needed to form a resulting intermediate unfolded molecule that subsequently misfolds. That is, it refolds into a misfolded toxic intermediate. However, an energetic photon or energy-rich neighboring substance must supply sufficient energy to enable this initial unfolding. It is often simply stated that the misfolded protein exists, with no source or precursor indicated. In such cases, it must be assumed that the misfolding takes place either during the protein molecule synthesis and subsequent flawed folding process or from a rare thermal stochastic event in quiescent solutions.

\section{Shear as a mechanical energy source producing "misfolded" protein seed}

This and previous papers in this series $[23,38]$ suggest that fluid shear processes within very narrow, tortuous path ECS within the brain produce shearinduced, conformation-altered amyloid monomers, designated $A \beta^{*}$. The conformations of these shearexcited molecules are proposed to be altered only to such an extent that the resulting unstable monomer molecular intermediate can initiate amyloid aggregation by reacting with other ground state amyloid molecules [39]. What is unexpected about this proposed shear-activation process is the relatively low energy needed to initiate the amyloid cascade reaction. This should not be surprising to amyloid chemists who are constantly plagued by amyloid aggregation when their monomer solutions produce aggregated protein if they not exceedingly careful in handling them. Nor should it surprise any amyloid researcher who has wondered at the loss of amyloid sample molecules during high performance liquid chromatography experiments (D. Teplow, private communication) [23, 38].

This proposed low-energy process is based on the carefully controlled spectroscopic studies of
Ashton et al. [25] where protein conformational changes in many different types of non-IDP proteins were observed with very small amounts of applied shear. Even more facile shear-induced conformational changes are anticipated with the more flexible IDP molecules such as $A \beta$, which have been observed but not published (L. Ashton, private communication).

Many anecdotal incidents have been reported revealing amyloid monomers, especially the $A \beta_{42}$ isoform, to be quite sensitive to liquid shear. Both the kinetics of formation [40] and the molecular structures of $A \beta$ fibrils derived from $A \beta$ [41] are different in the presence and absence of fluid movements leading to liquid shear in amyloid solutions. Amyloid aggregation induced by agitation, stirring, and other shear-producing fluid movement has been treated mostly as a hindrance in quiescent monomer experiments. This is because shear from handling these delicate solutions initiates and accelerates an aggregation cascade that creates high molecular weight fibrils and other aggregates, thus contaminating desired monomer solutions used in in vitro research experiments. On the other hand, in some cases, long lag times in amyloid aggregation studies or times for solubilizing amyloid monomer films have been shortened by stirring of amyloid monomer solutions or suspensions. Could this possibly lead to shear-induced amyloid monomer seed production? It is reported [42] that earlier experiments were not reproducible without stirring.

\section{An important mechanistic conundrum involving seeding}

Most in vitro amyloid research measurements have been conducted in the laboratory under quiescent conditions during the experiment, especially when dealing with the highly sensitive isoform $A \beta_{42}$. However, before these experiments were performed, solutions have been very carefully prepared by sonication, filtration, and other agitating purification steps before the experiments to rid them of solution impurities, mainly amyloid aggregates. In some cases, the solutions have been stirred to enhance the solubility of the amyloid monomer and filtered, sonicated, or centrifuged to remove these aggregates.

In the purification of the monomer amyloid solutions above, even if all of the dimers, trimers, and oligomers are removed, is it possible that the preliminary, possibly-shear-inducing handling generates shear that converts a small fraction of the monomers 
into a shear-altered monomer conformation seed that is not removed in the above solution preparation routine? Could that small, shear-altered monomer fraction of molecules be of similar conformations to those obtained by deliberate, reproducible shearing treatment, but acts as a very small reservoir of active seed in "quiescent" experiments during the lag time growth prior to the ultimate formation of oligomers, protofibrils, and fibrils? Could some of the monomers created by sonication retain a seed conformation that was responsible for their being present in an oligomer aggregation product? Could it be that these small numbers of shear-induced seeds are the cause of the long lag time and that there would be no aggregation without them? Would there be any amyloid aggregation without the formation of shearinduced, conformation-altered amyloid monomer or oligomer seeds that were not removed in the preparation or purification of the amyloid monomer? Could recent quiescent mechanisms proposed actually be from a seed originating from a shear rather than a thermal event? More importantly, could in vivo amyloid seeded aggregation be traced back to shear-induced events. Can such radical explanations be completely ruled out if the activated seed monomer has not yet been separated, isolated, or characterized? The experimental system suggested below is a possible way of exploring this problem.

An experimental attempt to answer these questions by Cohen et al. [40] by extrapolation to zero stirring revealed qualitatively different kinetics for stirred and "extrapolated-to-zero" stirring experiments. However, the aggregation in such "zero" stirring results may have been caused by seeds generated with different types of shear during the preparation of the quiescent solutions. For example, in Cohen et al. solutions were prepared by centrifuging them through filters, which force molecules through very small orifices, possibly causing extensional shearing of some monomers. Solutions were also purified by several rounds of gel filtration. It is difficult to avoid potential shear-inducing experimental situations, especially for $A \beta_{42}$. What are the different types of shear that possibly can change the amyloid monomer molecular structure?

\section{Different types of shear}

There are a number of amyloid-related diseases where the amyloid monomer presents in different proteopathic strains with different medical outcomes, e.g., prions $[11,12]$. There is growing evidence that

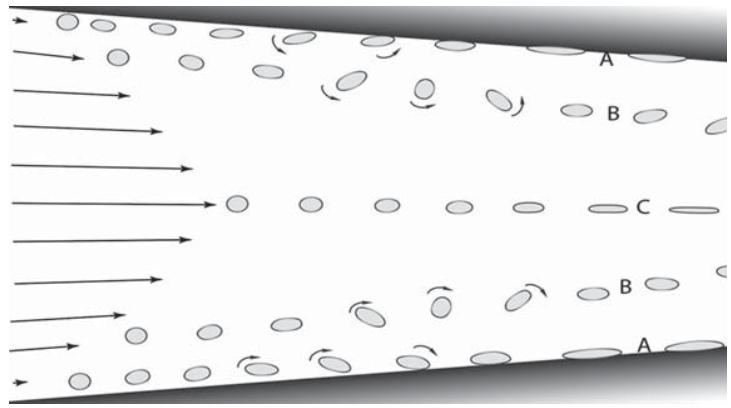

Fig. 3. Symbolic examples of three different effects of shear in a cross section of a narrowing flow path that generates both laminar and extensional shear, e.g., between two neighboring neuron surfaces (shaded black regions). Laminar shear is generated by diminishing differences in flow rates as one moves perpendicular to the wall surface. Molecules closest to the surface experience two forces, stretching and rotation. The latter forces a stretched end of the neighboring molecule to forcibly collide with and adhere to the wall (A), depending on the flow rate and geometry of the flow channel. Those molecules near the wall that are far enough away from the wall that they cannot collide with the wall freely tumble (B) while also oscillating in length, depending on their flexibility. Molecules in the center region have negligible laminar shear. However, as the distance between the flow-confining walls narrows, extensional shear is generated, as shown by the steadily lengthening and narrowing of the molecules shown in the figure. Left arrows represent liquid flow rates and molecular symbols represent stroboscopic freeze frames as a function of flow and time. Note the anticipated migration of B molecules away from the walls and toward the center axis because shear-stressed molecules tend to preferentially migrate from regions of high shear stress toward regions of lower stress [63].

this is due to different molecular conformations of the same amyloid monomer molecule. What causes these different strain conformations? Could it be the consequence of monomers being exposed to different ISF shear rates, shear types, and/or total absorbed shear energies in different parts of the brain?

There are two different types of easily predictable and comprehensible shear. These are laminar and extensional shear [23]. Both lead to stretching forces on molecules, which are most prominent in cases of pure extensional shear (Figs. 3C, 4). Laminar shear contains two force components in simultaneous molecular stretching and molecular rotation [43]. This rotation, if unhindered, leads to a cyclic oscillation of the molecule, the amplitude of the cyclic stretch depending on the shear rate. There is a rotating stretch-and-release, stretch-and-release, etc., cycle, during shear-induced free rotation (Fig. 3B) that, during many cycles, could cause major, conformational changes during extended, complex molecular motion, especially when the resulting conformation has a lower free energy. The molecules in Fig. 3A 


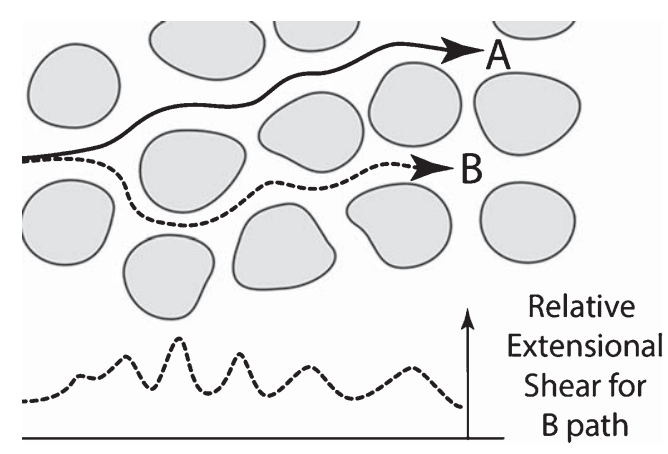

Fig. 4. High extensional shear (B) and low extensional shear (A) ISS flow paths through symbolic brain. Note shear oscillations in B path in graph and smaller oscillations in A path (not shown).

undergoing maximal laminar shear are those very close to or next to the planar (at the molecular level) surface such as a neuron and glial cell membranes. The maximum shear at this surface is caused by the immobile layer of fluid immediately next to the membrane. The rotation of a molecule very close to the wall probably causes a collision of the rotating molecule with that surface (Fig. 3A). The result of such a collision depends on the shear rate and attractive chemical forces between the rotating molecule and the surface. In Fig. 3A, the result is a sheared molecule adsorbed to that surface. Figure 3B shows free-cycling and oscillating molecules.

Extensional shear is produced when a flowing stream narrows between two flow-impeding objects, as shown in Fig. 3C, and rapidly accelerates in the central part of this stream as it enters the "narrows" between two flow-impeding objects. Figure 4 illustrates symbolically two different hypothetical paths through a hypothetical section of brain parenchyma. Each line represents the fastest flow paths through that particular region that both produce nearly pure extensional flow because they are always midway between the flow-impeding "neuron" objects, a highly enlarged segment is shown in Fig. 3. The B path in Fig. 4 has the higher extensional shear rates than the A path in Fig. 4 because it is, on average, a narrower path. In Fig. 4, note the oscillations in the bottom graph of the extensional shear along the B path. A defining characteristic of extensional molecular shear is that there is a greater acceleration on the leading edge of the molecule than on the trailing end as it enters the "narrows," thus stretching the molecule (observe the exaggerated elongation of $\mathrm{C}$ molecules in Fig. 3). Flow paths closer to the walls of the flow impeding objects undergo laminar shear containing smaller amounts of extensional shear.

\section{A potentially important role for extensional shear}

A recent publication [33] demonstrates that pure extensional shear flow that produces only stretching molecular forces causes protein aggregation through conformational remodeling in many different types of proteins and antibodies much more efficiently than with extensional shear originating from laminar flow. It is interesting that both shear rate and total shear (shear rate times time under shear) were important in this study in determining the protein aggregation results. The study showed that multiple exposures to extensional shear were necessary to cause aggregation of non-IDP proteins. This study also demonstrated that shear-induced surface hydrophobic protein character is an important driving force for such aggregation. It is anticipated that amyloid IDPs would be far more sensitive to pure extensional shear because of their greater conformational flexibility.

The above report implies to this author that stretching episodes with protein molecules ultimately expose hydrogen bonding sites on their protein backbones to their intramolecular surroundings, which can then more readily be transformed into different, lower free energy intramolecular hydrogen bonding patterns, ultimately giving rise to a cross beta conformation and thus ultimately initiating rapid protein molecule aggregation.

It is of interest that changing the composition of the $C$-terminal segment of $A \beta_{40}$ to $A \beta_{42}$ made a significant difference in a single-molecule force microscopy $A \beta$ dimer experiment [44]. It was a surprise that the $\mathrm{N}$-terminal segment, thought to be a disordered segment, plays a key role in the peptide interaction in the dimers. In these experiments, the molecule is perhaps stretched in the same manner in extensional shear experiments, especially if, in vivo, one end might be tethered weakly to a membrane.

It is this process of an intricate, possibly slow, conformational flexing attained through both extensional and laminar shear with extension and molecular rotation and oscillation providing a "roller coaster ride" on the free energy folding landscape that the author proposes during dimerization. However, this ride would be on a roller coaster that itself would be changing its shape underneath the rider molecule because of the influence of shear on that surface. It is difficult to illustrate such concepts in any graphical format.

Note that in the above single molecule force microscopy experiments, where the molecule is 


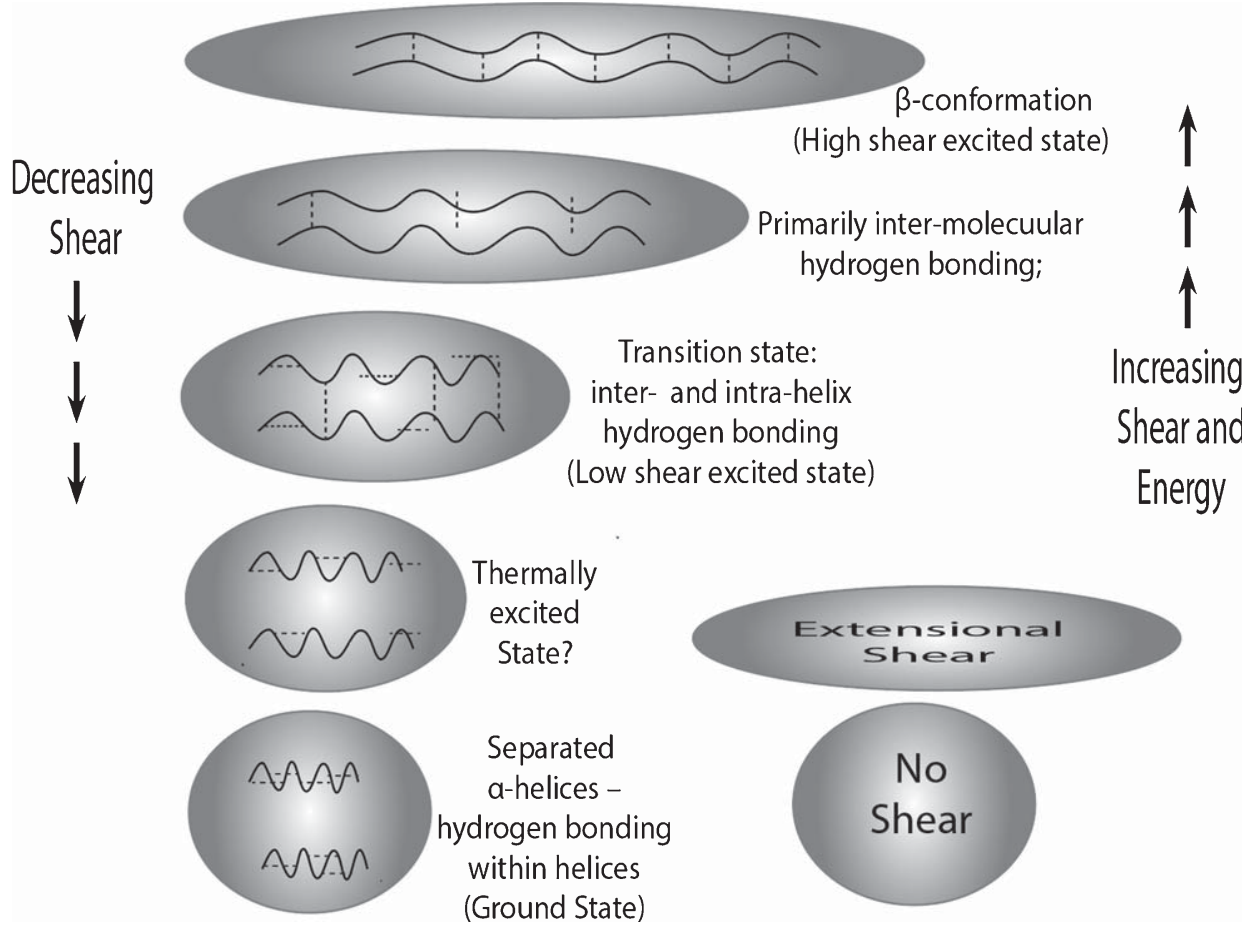

Fig. 5. Symbolic representation of the potential effects of extensional shear on a protein molecule, that is the distortion of the molecular shape from a spherical statistical random coil to an oblong shape. The very highly magnified wavy lines represent the protein backbones in two helical segments on the same protein that are in helical coil conformations in the un-sheared protein molecule at the bottom. With increased exposure to extensional shear, the coils and the hydrogen bonds within the helix are both increasingly stretched while the two molecular segments are brought closer together to the point that, in the transition state, there are hydrogen bonds possibly formed between the two segments. Further molecular stretching and narrowing of the molecule causes the two segments to form a predominantly beta conformation. The above is obviously an idealization and oversimplification of the actual situation in that there are many different orientations of alpha helical segments, etc. However, this illustration shows one possible type of intramolecular transition, from alpha to beta conformations that might be made possible because of shear. The process illustrated above may be a critical part of a pathway from quiescent amyloid monomer to a partial cross beta activated conformation that, with further shear oscillations and rearrangements, might act as a seed for the formation of toxic cross beta oligomers. As liquid shear energy is absorbed by the molecule, the molecule becomes shear-excited to different states, depending on both the shear rate and time the molecule is exposed, the total shear energy being equal to the shear rate times the time exposed.

mechanically pulled apart, that the force is not a random thermal event, but a deliberate mechanical, unidirectional force, much like that applied in a molecular fluid shearing event. Conformational changes induced in molecules undergoing these types of forces may be quite different from those taking place in quiescent molecules, where molecules undergo random Brownian collisions with its surroundings. Thus, increasing temperatures, for example, may not be able to promote the same kinds of stretching conformational changes as those achieved with extensional fluid shear.

If molecular stretching exposes the protein backbone to its surroundings, it may promote the formation of intramolecular backbone hydrogen bonding conformational transitions of the type illustrated symbolically in Fig. 5 that lead, in an extremely unlikely case, from two alpha helix segments on one molecule to a beta conformation in that same molecule. In Fig. 5, extensional shear elongates and narrows the molecule. This causes both stretching of helices that are oriented with the shear flow direction and forcing the stretching helices closer together, as shown in Fig. 5, which demonstrates only an ideal condition. In reality, the orientations of the initial helices will be not completely parallel and will thus probably be only in a partial favorable orientation as they approach one another during a transition state. As well, the amount of any new beta structure would be more limited than that shown in Fig. 5.

What is shown in Fig. 5 is the type of extensional shear in the entrance to a region of high extensional liquid shear halfway between two flow-blocking obstacles. Once having passed through "narrows" 
between the flow-impeding pair of objects, e.g., neurons, the extensional shear declines, as shown in the oscillations at the bottom of Fig. 4, and the stretched molecule relaxes and shortens. During this process, molecular reorganization can increase the degree of hydrogen bonding in beta-organized regions between neighboring protein backbone segments. An oscillation-induced transition that lengthens the region of beta sheet formation would be driven by the favorable net free energy change because of the greater thermodynamic stability of the beta sheet structure. Continued flow through the crowded brain region will present a series of flow-impeding pairs, as shown in Fig. 4, thus setting up an extensional shear oscillation for the fast-moving liquid segments in a flow stream.

With increased $A \beta$ concentration, there is a markedly increased probability of two stretched $\mathrm{A} \beta$ molecules, each with a more hydrophobic surface, being near neighbors as the molecules transit the "narrows" between flow-impeding objects. The developing beta configuration will expose more hydrophobic groups to hydrophilic solvent, encouraging aggregation of stretched molecules. Thus, extensional shear-stretching of amyloid monomers is proposed to significantly lower the activation energy barrier for the rate determining step in amyloid dimer formation, as illustrated in Fig. 1. The author speculates that this very low activation energy also may be caused by quantum tunneling [45] where there might be a synchronous making and breaking of hydrogen bonds in a sequential, zipper-like manner induced by the shear-induced stretching and relaxing cycle of the amyloid monomer molecule illustrated in Fig. 3B.

\section{Liquid shear during CSF and ISF flow within the brain}

Experiments have shown that within the rat brain both CSF and ISF are moving through different regions of the brain at different speeds [31, 46, 47]. CSF fluid flow rates within perivascular brain regions are proposed to be faster than ISF flow rates within the brain parenchyma.

However, any flow, however slow, through this flow-impeding parenchymal tangle must generate some level of liquid shear whose magnitude depends on the dimensions and shapes of the flow channels, flow-impeding objects, and ISF flow rates. This liquid flow is slow because much mechanical energy is expended in generating the significant liquid shear caused by the extremely small ISF flow channels as well as by the flow-impeding objects in that flow stream around which ISF must flow. Dissolved amyloid molecules trapped within the flowing liquid absorb some fraction of this mechanical energy because of shear forces. In doing so, they are deformed by these forces, especially near the walls of the flow channel where the shear is maximal. Maintaining this slow flow is critically needed to transport vital brain nutrients to and export waste products from the brain. Because of assumed very slow ISF flow, potential shear processes have been neglected or dismissed as important contributors to amyloid chemical reactions in the brain. Nevertheless, if there is fluid flow, however slow, there must be liquid shear, potentially affecting dissolved proteins.

Experiments involving the passage of liquid plug samples of proteins through very small bore capillaries in our laboratory [48] and in amyloid research laboratories with a high-performance liquid chromatographs containing connecting tubing identical to that used in our shear experiments report significant amyloid protein loss (D. Teplow, private communication). This probably results from the shear-induced transfer of protein to the inner wall of the connecting capillary tubing. The resulting protein coating, attributed by the author to shear at the wall [48] is metastable, but some protein dissolves back into quiescent solution when solution flow is stopped. Most of our early experiments were conducted with nonamyloid proteins.

However, recent preliminary experiments with $\mathrm{A} \beta$ solutions have indicated that shear-induced metastable $A \beta$ wall coatings are also present in a very small diameter capillary tubing flow system (Trumbore CN, Paik J, Vachet R, unpublished results). Preliminary results from these studies show that $A \beta$ is totally absorbed within the injection region and a very small amount of material, which is not identified as $\mathrm{A} \beta$ monomer, makes it through the capillary. However, if the flow is stopped for a period of 5-10 min, a much larger amount of non- $\mathrm{A} \beta$ monomer, previously surface-adsorbed material, is released into the restarted flowing stream. Repeated injections, followed by stopped flow periods, release smaller amounts of wall retentate in similar experiments. It may be that similar types of shear flow processes may have biological analogs in narrow ISF flow channels within the brain and that they may contribute significantly to amyloid chemistry. Further studies are planned. 


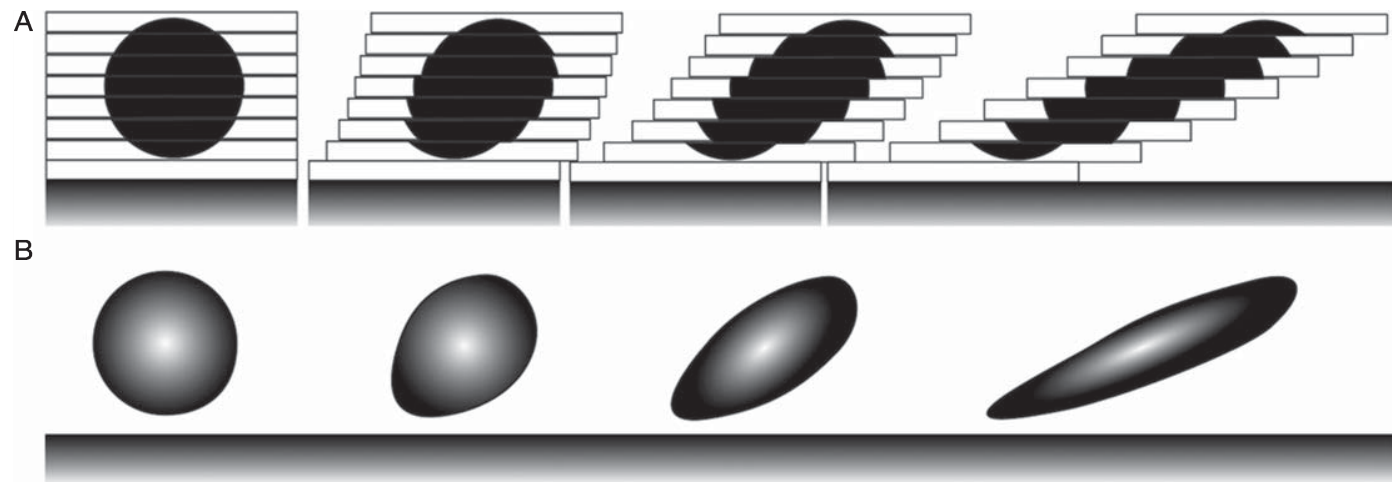

Fig. 6. Molecular model undergoing liquid laminar shear. The approximately statistically spherical random coil molecule on the left is subjected to laminar shear in a stroboscopic manner as it flows past a surface. This shear force is roughly presented in A by showing cross sections through the molecule center, with the molecule sliced in place before the seven layers containing these slices are each displaced to the right by a successively smaller rate with increasing distance from the surface, thus generating a laminar flow. B is an attempt to approximate the 3D molecular shapes resulting from the increasingly sheared molecule. The problem with this representation is that it does not take into account the chemical bonds that are resisting this mechanical shearing. The slices would be unable to stay in the same positions within their original flowing layer, the molecule would stop changing shape, and would start tumbling [43]. IDPs, being more flexible, might be easier to distort and might roughly approximate some of these shapes. With increasing shear, hydrogen bonds would be the first to break if the distortion became too great and if the shear rate were too fast.

\section{Molecular resistance to laminar shear}

Shear rate is directly linked to the rate of mechanical energy expended to promote shearing of the liquid. This energy is delivered by mechanical means through water solvent molecules to $A \beta$ in such a way that it mechanically (not thermally!) and directionally distorts the molecule, which resists this distortion, transforming mechanical energy into potential chemical energy needed to stretch or break chemical bonds. The total shear is determined by multiplying the shear rate (in seconds ${ }^{-1}$ ) by the total time (in seconds).

Long exposure to very low shear rates can deliver excess shear energy to a molecule subjected to such extended shear provided the sheared molecule is not able to shed all of this mechanical energy as heat back into the surrounding ISF solvent. That is, unless the stressed molecule is allowed to relax and shed its excess energy, it will continue to build up its energy content-if it is able to accept that energy. One situation in which it cannot is if the molecule has reached a limit to its being stretched at a particular shear rate. A rubber exercise band analogy is where individuals cannot stretch the band any further because of their strength limitations. Increase the trained muscle mass and one increases the stretch limit. If the rubber band's limit is exceeded, it breaks. The equivalent in this molecular case is breaking the weakest chemical bonds, usually hydrogen bonds, resulting in the first step in unfolding the molecule.
In Fig. 6, a molecular model is sliced up into 7 layers contained in 7 different solvent layers next to a wall where the eighth solvent layer adheres to the flow path wall and is immobile. The other 7 layers are each moving at different speeds. The difference in speeds between each layer pair defines the level of shear experienced by the object trapped in that layer. This neighboring layer speed difference, defined as liquid shear, diminishes slightly with each successive layer as one moves away from the wall. The upper part of Fig. 6 illustrates what changes in shape of the model one would expect just because of this speed difference as a function of time of flow.

However, molecules are not at all that flexible, but are joined, as is the rubber band (a rubber ball in this case) by chemical bonds that resist the shear and take up energy from the moving liquid to stretch the molecule. As with the rubber band, there is a limit to the amount of stretch these bonds can take, depending on the shape and chemical composition of the molecule. Thus Fig. 6 oversimplifies the situation but illustrates that the molecule will be exposed to mechanical forces that could readily cause a change in the molecular conformation, depending on the flexibility of the molecule and flow rate. IDPs such as many amyloid monomers are not only intrinsically disordered, they are inherently flexible and may come to somewhat resemble the shapes close to those shown in Fig. 6.

The distinction between shear and shear rate becomes especially important in such situations in 
which large amounts of energy are delivered very rapidly: think of receiving multiple gentle bumps versus one quick, concussion-inducing blow to the head using the same total energy. The rubber ball in the above discussion will be slowly distorted with enough force but can be torn apart by too much shear too fast. Scissors are also called "shears" for good reason, since they could theoretically be used to shear the rubber ball in preparation for the experiment in Fig. 6.

Theoretical calculations by others have demonstrated that, at equilibrium, macromolecules are in constant motion. Adding non-equilibrium shear forces to these calculations has been unwarranted (D. Teplow, private communication) because of the many complications, such as how to represent the many different combinations of laminar, extensional, and other types of shear not shown in Fig. 3. From a molecular point of view, for example, at high shear rates, a molecule may not be able to unfold in a natural way, i.e., the reverse of the way it was originally naturally folded. At low shear rates, it may be able to do so, but with some difficulty, very slowly, with gentle oscillations. An oscillating shear rate may be able to unwind and rearrange a particular conformation into an energetically more favorable state if it is in an oscillating laminar flow environment.

\section{Relaxation processes following shear pulses}

In a complex biological system such as the brain environment, the conformational future of any molecule is also very much dependent on its recent shear history and the nature and timing of the recovery from previous shear events. Suggestions have been made that CSF and ISF within the brain may be subjected to oscillating back and forth flow reversal with pulsing, non-equilibrium movements [29, 30]. If so, dissolved amyloids within the brain will be subjected to even more highly complex oscillating shear fields than otherwise anticipated from the above discussion.

The question arises, how fast and in what manner do shear-stressed molecules respond to reduced shear stress? A distorted rubber ball quickly recovers its shape. The shear-stretched $A \beta$ molecule $\left(A \beta^{*}\right)$ adsorbed on or absorbed within the membrane probably has a quite different relaxation time than that of a sheared $A \beta^{*}$ molecule contained within the confines of freely-flowing liquid ISF distant from a membrane. That is, recovery from non-equilibrium shear events probably will be faster in the liquid than on or within the membrane. This is why the extent of penetration into the membrane may be an important factor in determining the ultimate fate of a shear-excited $A \beta^{*}$ molecule originally a membrane neighbor. Thus, shear-induced tumbling, an important consequence of laminar shear, is likely to cause a tumbling $A \beta$ molecule next to the membrane to cause the extreme ends of that stretched molecule to more likely to make contact with the membrane. If the end segment is hydrophobic, this may tentatively anchor the $A \beta$ molecule to membrane surface hydrophobic elements.

Once contact is made, there are a number of types of bonds that can form between this extended, stretched molecule and the membrane surface with its polar and hydrophobic groups. If this is so, then the effects of shear next to the membrane wall may be more important than those taking place within flowing ISF in that the sheared molecule may not be able to relax and easily rejoin the ISF flow. It is then more likely to form a dimer or trimer or even ultimately a higher order oligomer such as $A \beta * 56$ [49] within the membrane. If the surface is that of a neuron membrane, higher order oligomers can form unregulated membrane channels that allow free movements of calcium ions, disrupting the neuron's function.

One of the problems with shear is its complexity, especially within the brain parenchyma. There is a report (G. Rose, private communication) of an industry that shipped a protein solution that aggregated when shipped in square bottles, but when shipped in round bottles, the proteins did not aggregate. The tortuous ISF flow paths within the brain extracellular space may generate other types of shear than the two main types previously discussed, laminar and extensional shear. Because of this complexity, it is certain that amyloid molecules will be exposed to, at minimum, complex mixtures of laminar and extensional shear and to shear rates that vary over time during their flow. Thus, modeling shear under both normal and disease state brain conditions will be exceedingly difficult. Nevertheless, the author has suggested an experimental system [38] and experiments below involving this system that allows investigations of laminar shear and especially encourages further experiments with another recently reported system that produces only extensional shear flow [33].

\section{Effects of pulsed flow rates}

A further potential complication involving in vivo shear is the effect of fluid flow pulse intensity. 
The movement within the brain of both CSF and ISF is reportedly controlled by a number of factors, including blood flow and the breath inspiration cycle [50] as well as cilia movement in brain ventricles [51]. One paper [52] raises the question of whether the increasing pulse intensity of the blood systole with age, which is reported to damage the blood-brain barrier due to hardening of the arteries, could also be a cause of AD? Does this increase in blood pulse intensity also result in faster maximum CSF and ISF flow, higher energy shear rates, and also higher energy excited $\mathrm{A} \beta *$ conformational states, which could also change shear-induced chemical products in response to increased shear pulses? Higher pulse intensity may produce an even further stretched excited state that exposes more otherwise hidden hydrophobic amino acid side chain groups, thus possibly leading to different dimer and oligomer products than those formed at lower pulse intensity and shear rates.

In considering consequences of increased CSF or ISF pulse intensity, one must translate pulse intensity into shear terms. This depends on the dimensions of the channels that confine the flowing liquid. The more these dimensions narrow, the higher the shear rate. The relationship between these two quantities is far from linear. For example, the shear rate in a circular cross section capillary is inversely proportional to the cube of the inner dimension capillary radius! Thus, when ISF flow channel dimensions get down to those estimated for parenchymal ECS [24], there need not be a very high ISF velocity to generate significant shear effects, especially with highly flexible, shear-sensitive IDP solutes dissolved in that fluid.

Thus far undiscovered shear-induced deposition of amyloid on ISF flow channel walls similar to those reported with non-amyloid proteins in stainless steel capillaries [48] could narrow the dimensions of these flow channels and significantly increase the shear rates, leading to a feedback amyloid deposition loop, especially if there is an increase in the flow rates from pulses described above and an increase in amyloid monomer concentration.

Peak velocity translates into peak energy rate delivery which translates into peak shear rates which translates into peak rates of molecular distortion, given fixed, or possibly channel dimensions that narrow with increasing age because of wall amyloid coating. The more energy that is made available in a given short period of time and not lost to thermal degradation, the more probable is a chemical transformation that is ordinarily prohibited by high activation energies. It is that sudden boost of energy that may overcome various activation energy barriers to deleterious brain chemical reactions in pulsing ISF.

A very large CSF or ISF pulse in a very short time, as might also possibly be experienced in concussion and traumatic brain injury could provide enough energy to break a number of protein hydrogen bonds, leading to a true partial unfolding of the amyloid protein rather than just a subtle conformational change. This kind of experiment, carried out in the laboratory, is one that this author believes will reveal very interesting results that may be applied to the studies of concussion and traumatic brain injuries in addition to gene expression changes induced by brain trauma.

\section{Competitive processes involving shear-excited states leading to dimerization}

One of the core CSF biomarkers for AD is the A $\beta$ isoform ratio, $A \beta 1-42 / A \beta 1-40$. In past mechanistic discussions by this author [23], the energetic, shearinduced conformational change product has been designated as $A \beta^{*}$ as in equation (1) below. It must be emphasized that $A \beta^{*}$ in brain fluids represents a wide variety of energy-rich molecules.

$$
\begin{aligned}
& A \beta+\text { shear energy } \rightarrow A \beta^{*} \\
& A \beta \rightarrow A \beta+\text { thermal energy }
\end{aligned}
$$

Equation (2) represents the relaxation of such a shear-induced excited state, with the release of thermal energy to the molecules' surroundings. This sequence of reactions, (1) followed by (2), is what happens when the $A \beta$ concentration is low, the solution molecule is no longer subjected to maximum shear, and the relaxation time of $A \beta^{*}$ is relatively fast. The net effect of these two reactions obtained by adding reactions (1) and (2) is that some or all of the shear energy has been converted into thermal energy and no net chemical reaction occurs.

In $\mathrm{AD}$, what is critical is what happens at higher concentrations of $\mathrm{A} \beta$ when reactions (3) and (4) can occur and compete with reaction (2). That is, when one or more chemical products are formed from the collision of a shear-activated $A \beta^{*}$ molecule either with a non-activated $A \beta$ or another shear-activated $\mathrm{A} \beta *$ molecule, thus preventing it from returning to its unexcited state. 


$$
\begin{aligned}
& \mathrm{A} \beta^{*}+\mathrm{A} \beta \rightarrow \text { Product } \mathrm{A}(\text { dimer })+\mathrm{x} \text { thermal energy } \\
& +\mathrm{A} \text { bond energy } \\
& \mathrm{A} \beta^{*}+\mathrm{A} \beta^{*} \rightarrow \text { Product } \mathrm{A} \text { or } \mathrm{B}(\text { different dimer }) \\
& \quad+2 \mathrm{x} \text { thermal energy }+\mathrm{A} \text { or } \mathrm{B} \text { bond energy }
\end{aligned}
$$

As the shear energy deposited in $A \beta$ molecules rises, it produces a variety of $A \beta^{*}$ shear-induced, excited molecule conformations that are more completely stretched or "unwound." The unwound molecule can still retain significant structure in the "still-wound" parts. However, with extremely sharp CSF spikes, such as those that might possibly be produced in concussions and traumatic brain injuries, it could be that very large shear energy injections might result in shear events causing true protein unfolding with multiple hydrogen bond breakage. If these events were to occur, resulting refolding could literally produce "misfolded" proteins because of the absence of nearby chaperone molecules [53]. Such misfolded proteins might possibly produce toxic products that could ultimately result in diseases similar to AD and other amyloid diseases, probably with unique chemical consequences producing pathology that differs from that of AD.

Under normal conditions, misfolded proteins are generally recognized and destroyed by natural processes [54]. However, such different, high-shear products that may indeed be misfolded proteins might not be recognized and cleared by these natural processes. In addition to dimerization processes between shear-stressed amyloids, there is the possibility of dimerization between sheared amyloid and other sheared, non-amyloid proteins.

Cross-seeding between $A \beta$ and tau has been reported in which seeds from one type oligomer induce aggregation involving the other molecule [9]. In addition, there is pathological evidence of molecular interaction between these two molecules leading to the formation of $A \beta$-tau co-aggregates [55]. One intriguing possibility is the dimerization of sheared $A \beta$ and sheared tau molecules, especially because of reports of the important role of tau in association with $\mathrm{A} \beta$ in $\mathrm{AD}$ etiology [56]. Could it be that a dimer of two sheared molecules, $A \beta$ and tau, is a possible catalytic intermediate in the formation of a different type of toxic $A \beta$ oligomer?

Chemical Equations (1) through (4) do not adequately express the energetics of shear reactions. Thus, symbolic energy level diagrams for all of these equations are presented in the Supplementary
Material for this paper. In addition, energy level diagrams are presented for equations not shown above that include similar competition reactions of $A \beta$ with membranes as functions of increasing $A \beta$ concentration.

\section{The relationship between shear energy and amyloid diseases}

In all of the mechanisms discussed above, the amount of "shear energy" in these equations and diagrams absorbed from the liquid shear process by $A \beta$ is dependent on the liquid shear rates in specific brain locations where mechanical liquid shear energy is transferred directly to the $A \beta$ molecules. Because of the many different kinds of shear events in the brain, the actual amount and types of shear energy transferred to the $A \beta$ molecule in any specific brain ISF flow channel location and shear condition probably varies widely. For the same reason, the amount of time the shear-excited $A \beta$ molecule has spent under shear and is able to relax probably also varies widely. Thus, as it flows in the ISF, an individual $A \beta$ molecule is continually being distorted and relaxed in a manner that is totally dependent on the "shear map" that lies behind and ahead of it in its glymphatic pathway journey through the brain. Does this also mean that the resulting shear-induced chemical reactions are different in different paths? We lack experimental answers to this question.

It seems reasonable that shear-induced amyloid aggregation products are probably both total-shear as well as shear-rate dependent. This is because both the accumulated amount of shear and the intensity of the shear rate will determine what kind of side groups, e.g., hydrophilic, hydrophobic, and ionic, and main backbone hydrogen bonding sites, become exposed in the shear-distorted $A \beta^{*}$ molecule. A large number of permutations and combinations of stressed monomers should result in a variety of shear-stressed, aggregated amyloid monomers, whose formation and relaxation rates will also be dependent on the environmental shear variables contained within the brain shear map.

Thus the conformations of the shear-induced products may also differ in different parts of the brain. The age of an individual brain may also help determine the nature of this shear environment. If different amyloid monomer conformations lead to different disease courses, as has been the case with the different strains of prions [12], then the same may be true of the different unstable and diverse shear-induced templated $A \beta$ 
oligomer products formed from unstable and diverse monomers and dimers. Such dimers may well act as seeds that either lead to toxic oligomer formation or catalyze an amyloid cascade leading to protofibril formation or both.

Consequently, the author suggests that the amyloid aggregation and resulting disease states that this shear-excited protein molecule initiates also depends on the brain shear map. If different strains of prions cause different symptoms, then it is probable that different shear-induced amyloid monomer strains are produced in different parts of the brain at different times, possibly leading to different diseases and disease symptoms, e.g., AD, Parkinson's disease, tauopathies, etc. [4].

Nearly all of the experiments in amyloid chemical research have been conducted in quiescent solutions. A number of experiments have been conducted under conditions in which it is possible to contrast qualitative results under both shear and non-shear conditions. However, only a limited number have been conducted under well-defined and repeatable experimental conditions. In two particular studies $[40,41]$, the results have been significantly different, thus leading this author to repeat his previous urgent appeal [23] to scientists to repeat critical quiescent amyloid experiments under shear conditions such as those outlined in supplemental material of the second paper of this series [38] as well as the more limited set of recommended experiments outlined below.

The reports of Liu et al. [13] and the related work of the Spires-Jones group [57] reveal that different parts of the brain parenchyma, those surrounding senile plaque and those far from plaque, produce quite different biological and neurological oligomer toxicity and synapse viability. These differences may be related to different shear types and energies and will be discussed in more detail in the next paper in this series. The potential importance of the ISF-flowimpeding property of senile plaques will be discussed in hydrodynamic terms in that paper.

\section{Spontaneous thermal reactions in quiescent solutions versus shear reactions?}

The potential role of shear has been emphasized in the above discussion. The question arises, what could be the driving force in quiescent solutions to form similar types of activated $\mathrm{A} \beta *$ molecules as are postulated in shear situations? First, one must remember that the crowded conditions within the ISF flow channels may increase the free energy level of the $A \beta$ within the ISF.

The term quiescent does not reveal the many different types of energetic molecular collisions responsible for the thermal chaos in such solutions. The energy distribution in a quiescent solution might be described by a Boltzmann-like energy distribution for liquids, where at any given time there are a small number of solvent molecules with rather large kinetic energies and a much larger number of molecules with much lower energies.

A dissolved $A \beta$ molecule is continuously being randomly bombarded by water molecules and perhaps other molecules on all sides. When highly energetic water or other molecule collides with $A \beta$ in just the right manner to distort it away from one of the most highly populated conformational states, this thermally excited $\mathrm{A} \beta$ may be vulnerable to dimer formation. However, if the $A \beta$ concentration is relatively low, and some of the solvent molecules surrounding $\mathrm{A} \beta^{*}$ are of lower energy, the molecule may relax quickly, lose thermal energy and continue sampling the normal populated lower energy $A \beta$ conformational states [58]. On the other hand, if the $A \beta$ concentration is increased, the probability becomes much higher of a productive collision of two excited $\mathrm{A} \beta$ thermal states, possibly creating a low-energy excited dimer state.

Figure 7 is a speculative diagram of what might possibly illustrate the difference between a random thermal spike-induced and a shear-induced dimerization of $A \beta$. In the upper solid curve (a) of Fig. 7 is depicted the smooth transfer of shear free energy from the sheared surrounding solvent to a single $\mathrm{A} \beta$ molecule to elevate its free energy to that of shear-state $A \beta^{*}$, which has just enough energy to react with either an unexcited $A \beta$ molecule or with another $A \beta^{*}$ molecule to ultimately form an $(A \beta)_{2}$ dimer. In the more random, energy-spiked lower (b) curve is a crude representation of the transfer of smaller amounts of energy to the $A \beta$ molecule, with a rare, very high-energy Brownian motion pulse that contains sufficient energy to elevate the $A \beta$ to a low-energy thermal A $\beta *$ state that is sufficiently energetic to form a dimer when reacting with another $A \beta$ molecule at high $\mathrm{A} \beta$ concentration.

The question raised by the above discussions that the author believes needs to be answered by the amyloid research community is: Are the dimers and successive aggregation products formed by the shearinduced and thermal-induced processes identical? For example, are the two different-energy excited $A \beta$ 


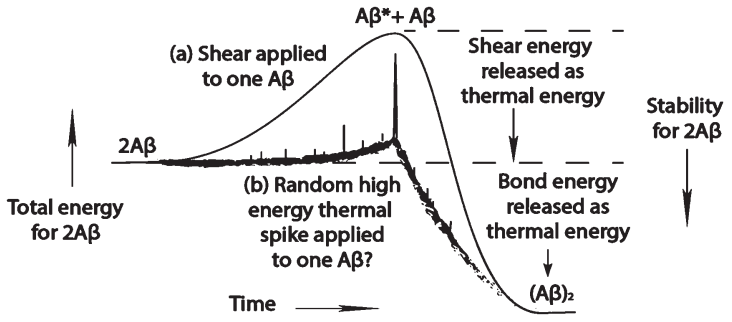

Fig. 7. Speculative diagram of free energy uptake that leads to the formation of an $A \beta$ dimer as a function of time by one $A \beta$ molecule: (a) during a liquid shear event; and (b) in a quiescent solution that has a stochastic high energy event that energizes the molecule by severely distorting that molecule.

molecules actually the same because, even though they were formed by two different processes and have different energies when they were first formed, they both may have lost these different energies via thermal relaxation processes to wind up at a common energy state $A \beta^{*}$.

Figure 8 represents: (a) an $A \beta$ molecule absorbing enough thermal (Brownian) energy to make a significant $A \beta$ conformational change to form a thermally excited $A \beta$; (b) the absorption of a slightly higher shear energy to create a roughly equivalent conformational change; (c) a very high amount of shear energy deposited into an $A \beta$ molecule, say from a blow to the brain that causes high-energy liquid shear in some part of the brain; (d) a fast loss of energy via shedding of thermal energy gained from absorbed energy events such as in (b) or (c), followed by a pause resulting in a metastable conformational state that is identical to that found in (a) or (b) events. The type of energy-shedding shown in (d) could produce a reaction of two of these energized molecules to give the same products. The (b) and (c) energy absorption events could shed energy in such a way that the products formed from $A \beta$ molecules energized by thermal as well as shear mechanisms might produce the same products. On the other hand, these high energy $A \beta^{*}$ molecules may not yield back to their environs their excess shear energy as depicted and produce quite different products. The author believes that this uncertainty can be resolved by experiments that are outlined below and in the supplemental materials of the second paper in this series [38].

The higher the energy events, the more likely may be energy-shedding events such as (d) in which the excess energy is shed as thermal energy, possibly in successive steps as shown in Fig. 8. However, there may be many metastable energy levels that lie in between these very high- and low-energy levels. This

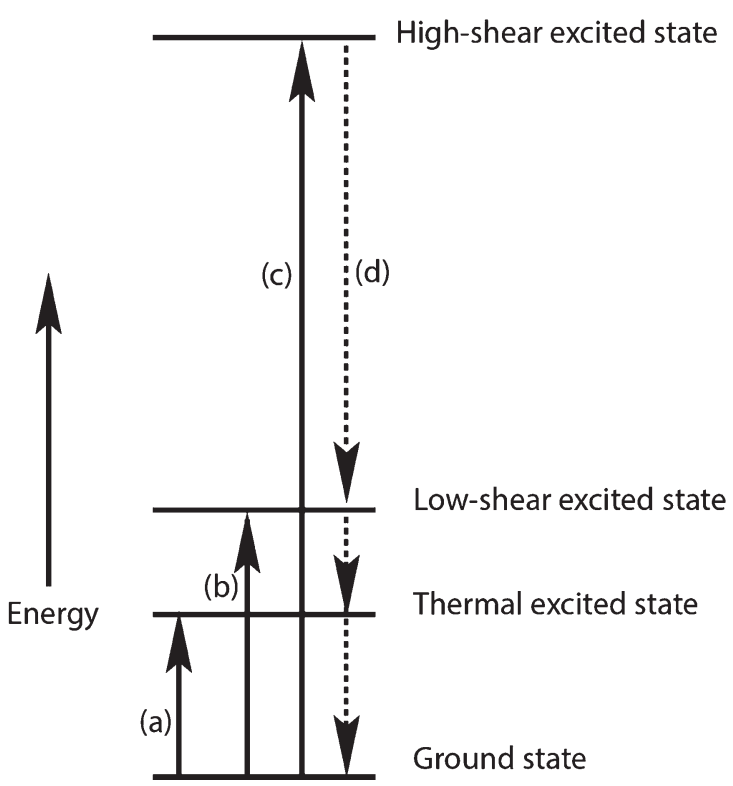

Fig. 8. Greatly oversimplified diagram of thermal and shear events that add energy to an amyloid monomer followed by thermal energy transfer to the surroundings. Dashed lines indicate uncertainty about the rate and timing of this energy loss.

allows a wide range of shear energy to produce products that may or may not be different from those produced by low-shear energy or thermal energy events.

If it is found that there are different shear-induced products produced by different shear energy $A \beta^{*}$ reactants, the author speculates that such types of different chemical products may be responsible for a wide range of amyloid disease states. The author believes that the above speculation is subject to experimental verification using the experimental system in experiments proposed in the previous paper in this series [38] and below.

Because $A \beta$ is a relatively "flat" free energy IDP, that is, with many different possible conformations possible at room temperature, it is a highly flexible molecule. The author questions whether the some of the conformations accessible under these thermal conditions are the same as those accessible under certain shear conditions. In particular, are those under extended extensional shear conditions the same? In extensional shear, a molecule will continually be stretched, whereas any stretch of a molecule under thermal conditions will be usually quickly countered by a relaxation in the opposite direction. Phrased another way, does the deposition of directional mechanical energy create a uniquely different conformational outcome from a random 
thermal event? A macro example might be comparing a seaweed patch during an incoming tide with the same patch in a quiescent ocean that is being heated. The seaweed, as well as the floppy parts of an IDP, orients preferentially with the flow whereas heating does not.

\section{Laboratory models for studying brain shear energy and various types of shear}

Thus, one of the most important questions this paper poses is what role does variable shear energy play in $A \beta$ aggregation within the brain? Brain ISF shear is highly variable and therefore generates highly variable $A \beta *$ shear-created energy states. $A \beta$ molecules flowing through brain flow channels are subject to a wide variety of total shear and shear rates, not to mention shear effects involving the many flowimpeding molecular species that block the $A \beta$ flow path, such as the structural elements of the perineuronal network [59]. Consequences of this flow may be adsorption of $A \beta^{*}$ on the membrane and aggregates derived from this shear-excited state. How can one model in the laboratory provide such a wide variety of shear consequences?

In capillary tubing laminar flow, liquid shear is highest immediately next to the capillary wall because the water layer immediately next to the wall, which wets the wall, is immobile. Its flow rate is zero. The water layer next to this layer moves at a comparatively high velocity, i.e., when compared with zero wall layer velocity. However, moving perpendicular to the wall, each successive liquid layer moves at a faster flow rate. However, the difference between each successive layer flow rate and that of its neighbor is successively smaller until, in the center of the capillary, the difference between neighboring layer flow rates is nearly zero. Therefore the shear rate in the exact center of the capillary is near zero. Shear rate is proportional to the difference between the flow rate of one layer and its next layer neighbor. Thus, the maximum laminar shear rate is maximum at the capillary wall. This means that if there is shear-induced conformational change promoted by laminar flow, it is maximum at the wall.

If shear-induced $A \beta^{*}$ is the precursor to aggregation, what are the flow-induced consequences of this within the brain? Although the flow channels in the brain are undoubtedly quite different, it is proposed that some similarity with capillary processes allows capillary shear processes to be a rudimentary first step in modeling the brain flow-induced amyloid chemistry. The advantage of the capillary is that the shear rate is near zero at the center of the capillary, which will be a distinct advantage in the capillary system proposed below for use in shear experiments.

The capillaries used in our early experiments [48] had a uniform inner diameter. Therefore, they generated only laminar shear, which does have a component of extensional shear. However, the recent experiments of Dobson et al. [33] report a system that generates only extensional shear. Thus, these two techniques provide an opportunity to study both laminar and extensional shear in model systems. Mixtures of these two types of shear can also be generated in the narrowing diameter system illustrated in Fig. 3. Such a geometry models an ISF flow stream approaching two rounded objects such as two neurons or a neuron-astrocyte pair.

\section{CRITICAL SUGGESTED EXPERIMENTS}

In the second paper of this series [38], there were a large number of proposed experiments listed in its Supplementary Material that used the proposed capillary tube system in the study of liquid shear processes involving amyloid monomers and oligomers. Of these many experiments, one stands out in particular dealing with the major topic of this paper-the role of variable shear energy in relation to various amyloid diseases. This is the experiment in which the experimental stainless steel capillary system sketched in cross section in Fig. 9 below is used to study the effects of variable shear and shear rates. Briefly, a cylindrical liquid sample (dashed lines in A and B in Fig. 9) is injected at time zero (see inset graph of "Detector Signal" versus "Time") into a flowing aqueous mobile phase and is stretched and distorted by laminar flow as a function of time, as indicated in the upper part of Fig. 9 ("A") at time "a," and at a later time " $b$ " in the lower "B" profile. At time "b," the time at which the front tip of the sample reaches the path of the light source, the detector signal (sample absorbance) increases suddenly, as indicated in the inset graph, peaks, and then exponentially decays. Further system details are contained in reference [38].

An exponential decay graph shown in the Fig. 9 inset is an indicator that amyloid sample molecules had very little time to diffuse. If these molecules do diffuse significantly, the curve will consist of a peak and shoulder [38]. The following recommended experiments should be carried out under exponential decay conditions outlined above. If a shoulder 


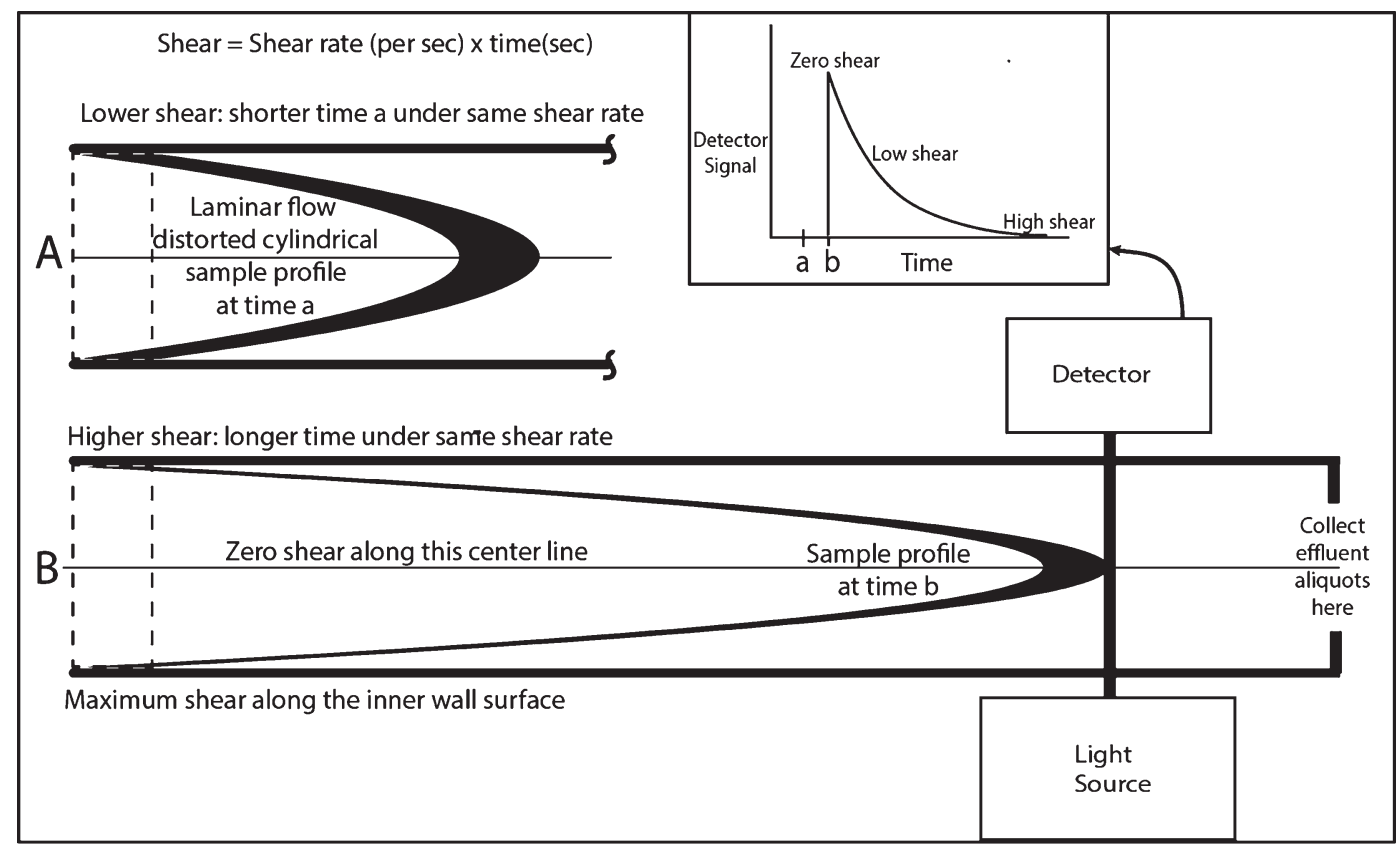

Fig. 9. Cross section of an empty capillary tube used to generate laminar-sheared amyloid solution aliquots that are exposed to increasing amounts of shear and to higher shear rates. Cylindrical amyloid sample (dashed lines on extreme left) is distorted by laminar mobile phase flow toward the right in which the flow rate is zero at the wall and maximum along the center line. The inset is a graph of light absorbance (detector signal) by the sample as the developing sample profile passes through the region lit by the ultraviolet or other light source. The A image is an early profile at time a and B is the sample profile at time $\mathrm{b}$ as it just begins to absorb the light, suddenly increasing the detector signal. At the tube outlet in B on the extreme right, small aliquots of the emerging sample are taken for experiments testing the effects of increasing shear and shear rate. Assuming negligible diffusion of the amyloid molecules, the first aliquot sample has near zero shear. Subsequent aliquots have been exposed to both increasing shear and shear rates. To study only the effect of shear rate, one could shorten the tube length, keeping the flow rate the same or increasing the flow rate in the same length system. There is no pure extensional shear along the center line because the capillary diameter is constant. If it narrowed from left to right, extensional shear would occur there.

is detected, purely exponential conditions can be obtained by increasing the mobile phase flow rate. Recognize that at higher flow rates, and therefore higher shear rates, there is a higher probability of amyloid aggregation at the dashed lines wall location where the original sample resided before flow began and there is a great need for cleaning the tube thoroughly with mixes of several different alcohols or nitric acid before performing another experiment using that same capillary.

The effluent exiting on the right hand side of the capillary in Fig. 9 is collected in small aliquots. The first aliquot contains sample from the center of the capillary that has been subjected to near zero laminar shear, depending on its volume. Subsequent small aliquot samples will have been subjected to increasing shear and shear rate.

Since total accumulated shear is equal to shear rate $\left(\right.$ seconds $^{-1}$ ) times time (seconds) [60], subsequent samples exiting the capillary tube with time will have been closer and closer to the wall and thus will have been subjected to not only increasing shear rates, since the maximum shear rate is found at the capillary inner wall surface, but also to higher total shear because they exit the column at a later time. Thus, the final aliquots have been subjected to not only very high shear rates, but very high total accumulated shear, greatly enhancing shear effects. If there is a desire to lower the total shear but keeping the same shear rate, one can reduce the length of the capillary.

Aliquots can be collected from the above experiments that have been subjected to a wide range of shear rates from near zero to very high values. With skillful engineering and experimental design, the effects of shear can be tested on a range of subsequent in vitro or in vivo experiments by adding aliquots in the shortest possible time to quiescent or possibly flowing test solutions containing test reagents or biological species. Aliquot samples exiting the column should make immediate contact with either quiescent $A \beta$ solutions to study seeding effectiveness or with a biological system of interest for 
toxicity studies. These results should be compared with aliquots that are not added to test solutions until after variable delays. In this manner, it may be possible to detect the decay times of short-lived monomer excited states if they have comparatively long lives. More likely dimers will be formed upon any contact between quiescent monomer or capillary $A \beta^{*}$.

Given the experimental design above, the concentrations of sheared molecules may be too low for accomplishing certain experimental goals. Increasing the concentrations also increases the incidence of bimolecular reactions among sheared and nonsheared molecules, which may be the desired goal. Studying the effects of diminishing amyloid monomer concentrations could possibly reveal the relaxation characteristics of different single molecule shear-excited states. Under certain conditions, instead of having plug samples, sample solutions in a recycling loop could be studied at various speeds. It will be difficult to study selected different regions of the shear stream, especially those regions near the wall where the shear rates are the largest. However, since low shear rates are apparently important within the brain, it may be possible to increase the experimental flow path width and increase the flow rate to achieve larger sheared volumes at a given shear rate. Oligomer and protofibril formation will probably occur at high $\mathrm{A} \beta$ concentrations.

Physicochemical studies can be carried out spectroscopically in stopped flow experiments in which the mobile phase flow carrying the distorted sample is suddenly stopped at different times, parking different parts of the sample that is contained within the detector zone at various times. This parked sample, whose shear history is known, now can be studied as a function of time to observe shear-excited molecules relax and to follow the kinetics of any shear products that can be observed as sheared molecules diffuse and react. Studies can be made with circular dichroism, UV, Fourier Transform IR, of parked samples. Sample amyloid monomer concentrations can be increased to promote aggregation of sheared molecules within the parked sample. Extrapolation to zero concentration can be studied to tease out single sheared molecule behavior. Various types of mass spectra of capillary effluent can be used to study effluent intermediates and reaction products. The effluent could possibly be used to form micro-droplets [7] and carry out single molecule experiments, especially seeding variations. Many variables can be tested using this simple system, including shear rate, total shear, monomer concentration, temperature, adsorption to surfaces, etc.

However, in view of the importance of seeding in amyloid science, one of the most important research goals probably should be to study the differences among oligomer products produced under: a) distinctly shear-only conditions, e.g., continuous feed of sample solutions through the capillary; b) seeding of quiescent solutions with exogenous shear-only produced products, c) seeding of quiescent solutions under just quiescent conditions. If products differ, this will demonstrate that it is quite important to continue studying the effects of shear. If the products are all the same, there is the possibility that either: a) all products have their origins with a shear process; or b) the sheared excited state relaxes very rapidly to a very low energy excited state that can be reached by some high thermal energy process.

The cross sections in Fig. 9 are described as intended to depict capillary flow, but could well represent flow in parallel plate flow, which could mimic flow between neuron and other glial membrane surfaces. By varying the end flow path thicknesses of these plates, one could reproduce this geometry to emphasize the importance of extensional shear. Flow geometries producing shear other than laminar or extensional could be explored to mimic tortuous ISF brain path environs.

\section{Potential applications in drug development}

Conventional stopped-flow techniques [61] could be used in combination with the above capillary tube system in which capillary effluent is mixed with another flowing fluid containing test molecules that react with —or do not react with — the stressed amyloid monomer. Test molecules could be potential anti-amyloidogenic drugs, chaperone molecules or the many small molecules that have been tested in an attempt to prevent the amyloid cascade reaction [19]. Antibodies against new shear-induced products could be tested. Alternatively, and perhaps even more importantly, these same potential reactants above could be added directly to the mobile phase, recognizing that they might be affected by the in vivo shear environment themselves and react with the amyloid monomer in a different manner. The latter would be a more realistic situation since all drugs and antibodies would be subjected to shear within the AD patient! For example, it has been shown that antibodies are shear sensitive [33]. 


\section{A warning!}

In any experiment with amyloid monomers or other shear-sensitive amyloids, it is important to realize that one needs to use great caution if these chemicals are handled with micro- or other very narrow-gauge syringe needles. As warned previously [38], at surprisingly low flow rates, these needles, and capillaries, can generate enough shear to initiate coating of the inside of these needles with a metastable protein coating, so that the delivery of "known" quantities of shear-sensitive amyloid monomers, especially $A \beta 1-42$, may not be accurate or reliable because of aggregation losses to the wall and subsequent "bleeding" from the inner surface of the needle and contamination in subsequent experiments. This phenomenon is concentration dependent. Therefore, extreme care should be taken in minimizing the speed with which one pulls up and pushes down the sample plunger while using a microliter syringe with amyloids, especially at high concentrations, e.g., tens of micromolar. It would be advisable to experimentally determine whether there are losses during variable sample delivery speeds.

This warning could be exceedingly important for any experiments in which amyloid monomers are being transferred or delivered. Even if there is not a shear-induced plating out of amyloid on the inner needle wall, there could be enough gentle shear to shear-alter the amyloid monomer and form soluble oligomers at a distance from the wall, which then could possibly act as an active shear-induced seed within the delivered liquid!

For those experimenters who engage in electrospray mass spectrometry, there have been problems with amyloids clogging the entry tip [62]. Any experimental device in which the amyloid solution is forced to flow through a very severely narrowing passage could induce amyloid aggregation because of high extensional shear during sample passage. Selective molecular weight filters could possibly induce aggregation, depending on hole geometry and flow rates. Extensional shear will exist in any passage that narrows significantly over its length.

\section{SUMMARY AND CONCLUSIONS}

It is proposed in this paper that liquid shear processes initiate the conversion of mechanical energy generated by fluid flow through highly restricted flow paths in the brain into chemical energy to form a mechanically distorted, chemically excited, $A \beta$ monomer $(\mathrm{A} \beta *)$. This excited state probably has a conformation that is different from any thermally excited monomer or group of monomer molecules because it is mechanically distorted. For this reason, it is proposed that shear-induced chemical products evolving from this shear-induced seed molecule may be different from those found in experiments using quiescent $A \beta$ solutions. Furthermore, such shear-induced brain products are proposed to be responsible, at least in part, for the neurotoxicity in the brain resulting in amyloid disease.

The energy of $A \beta^{*}$ is dependent on the brain shear map location and therefore is variable, with potentially different products and therefore potentially different neurotoxic effects in different regions, possibly resulting in different amyloid diseases. The lifetime of this excited state is limited if the shear is removed or lowered. Reactions of other amyloid monomer molecules with this state is highly concentration dependent and therefore age-related if this shear-excited state is involved in $\mathrm{AD}$.

The two types of mechanical liquid shear energy considered in these papers, laminar and extensional, should generate through liquid shear sufficient amounts of mechanical energy to cause subtle, but critical, conformational changes in amyloid monomers in certain parts of the brain that then initiate amyloid aggregation reactions. Extensional shear flow has been shown to cause many different types of protein to aggregate. This is caused by conformation changes that expose hydrophobic side chains normally buried within the interior of the protein on or near the protein surface to aqueous solvent. Therefore extensional shear is a likely inducer of amyloid type aggregation within the brain.

The potential importance of seeding is stressed in this paper. Shear-induced conformational templating, much like the transformations induced by prions, apparently arises from amyloid monomer seeds that induce conformational change in other amyloid monomers that come in contact with these seeds. It is proposed in this paper that shear is a driving force in creating oligomer seeds. Once these seeds are formed, aggregation proceeds at a rapid pace in comparison with that observed in the lag phase in quiescent solution aggregation. The question is raised whether small quantities of undetected shear-induced seeds are responsible for the long lag times in the formation of detectable amyloid fibrils in quiescent solutions.

The author proposes that there are two types of brain parenchymal seeding taking place in vivo, one 
on the neuron surface, the other on proteins anchored in the membrane surface, but at a short distance from the neuron surface. Both types are proposed to originate in flowing ISF, with the highest shear rates at and near the neuron surface. Because of like bonding attractions between shear-stretched monomer and the membrane surface, the seed is the adsorbed stressed monomer, which has a limited lifetime in this state, and loses its excess shear stress energy if it does not encounter another monomer to form a membranebound dimer. On the other hand, the lower-energy sheared solution monomer, not in direct contact with the membrane surface, is proposed to interact with branched membrane-anchored proteins, forming a protein-bound seed, which then interacts with and retains monomers in flowing ISF to form senile plaque [23].

The mechanism proposed for the transition from the $A \beta$ amyloid monomer to a cross-beta structure oligomer has been the subject of controversy. It is proposed in this paper that this transition can be greatly assisted by extensional shear that both stretches and narrows the $A \beta$ molecule causing segments of exposed protein backbone hydrogen bonding sites in neighboring shear-distorted alpha helices to smoothly switch from alpha helix to beta sheet bonding segments. The complex motions induced by oscillating shear can assist in promoting this transition from primarily alpha helical to beta sheet conformations, possibly in many short conformation-altering steps.

The case can be made, following by analogy the argument by Stone et al. [52], that the intensity of brain liquid shear may increase with age. With increasing age and $A \beta$ concentration and stiffening of the arteries, the blood pulse-and thus perhaps also the CSF pulse strength also-increases. Does the average energy of shear-excited $A \beta *$ also increase with age because of this? Do the chemical products also perhaps change during collisions of higher shear energy $A \beta^{*}$ molecules, forming chemically different dimers than those formed from lower energy $A \beta^{*}$ molecule collisions? Thus, a related critical question is: Does increasing shear energy in general change the conformations of the $A \beta *$ neurotoxic oligomers and therefore create new molecular strains with different toxicity and etiology of AD with age?

Summing up, this paper raises one overarching question. Does shear-induced amyloid monomer seed formation followed by templated conformational changes leading to amyloid aggregation in the brain answer the critical question that has eluded all attempts to identify the origin of at least some of the "misfolded" amyloid monomers? Until this and the other related questions raised in this paper are experimentally addressed, this author believes that shear cannot continue to be ignored as a critical variable in the etiology of $\mathrm{AD}$. The author suggests that the answers to many of the questions posed in this paper can be obtained in the laboratory using the capillary system suggested above and in the second paper in this series [38] as well as a recently reported extensional shear system [33].

Although CSF flow rates are being made around arteries entering the brain, it has not been possible to measure parenchymal ISF flow rates within the brain. Estimates of parenchymal channel dimensions have not been confirmed. However, it is believed by this author that sufficient experimental evidence is available to imply that CSF and/or ISF shear in different part(s) of the brain must be present and that shear, at least in part, must be responsible for some amyloid monomer aggregation processes and toxic oligomer formation, especially near membrane surfaces.

Although this paper focuses on the effects of shear on amyloid-related molecules, shear may also be affecting all soluble and wall-adsorbed or embedded molecules, including those that might possibly react with other sheared proteins. Shear may well affect the nature of transition states and outcomes of many brain chemical reactions.

It is this author's opinion that the current amount of brain amyloid shear research is insufficient, but nevertheless provides enough evidence to urge the amyloid research community to drastically increase research activity in this field. The author has suggested in this and in the previous paper in this series [38] an abundance of experiments that are available to those who are interested in exploring this important new area of amyloid research.

Although the papers in this series have focused on $\mathrm{AD}$ and $\mathrm{A} \beta$, there is every reason to expect that the above proposals regarding shear-induced conformational changes leading to amyloid aggregation probably also apply to many other amyloid-related diseases, e.g., Parkinson's, tauopathies, Lewy body, and other dementias because of the many similarities among these diseases [4]. This author speculates that the variable shear energies available in different parts of the brain and at different ages and physical conditions may be the cause of the wide variety of amyloid diseases and that the amyloid research community should initiate shear studies on mixtures of amyloid monomers, chief among these being mixtures of $A \beta$ and tau. 
The next paper in this series will justify reports of oligomer formation and the inactivation of synapses neighboring senile plaques and elsewhere [57] based on shear concepts outlined in the papers of this series, induced by the ISF flow disruption caused by the large plaque particles and small flow-impeding neuron projections.

The author challenges amyloid researchers to experimentally test these and many other speculations made in this and the previous papers in this series. The past basic research has thus far yielded no successful clinical drug trials. Is it not time to try a different approach?

\section{ACKNOWLEDGMENTS}

The author gratefully acknowledges the editorial help of David Teplow, Robert Suter, and Elinor Thomforde.

The author's disclosure is available online (https:// www.j-alz.com/manuscript-disclosures/17-1003r1).

\section{SUPPLEMENTARY MATERIAL}

The supplementary material is available in the electronic version of this article: http://dx.doi.org/ 10.3233/JAD-171003.

\section{REFERENCES}

[1] Joshi P, Chia S, Habchi J, Knowles TP, Dobson CM, Vendruscolo M (2016) A fragment-based method of creating small-molecule libraries to target the aggregation of intrinsically disordered proteins. ACS Comb Sci 18, 144-153.

[2] Cremades N, Dobson CM (2018) The contribution of biophysical and structural studies of protein self-assembly to the design of therapeutic strategies for amyloid diseases. Neurobiol Dis 109, 178-190.

[3] Hardy JA, Higgins GA (1992) Alzheimer's disease: The amyloid cascade hypothesis. Science 256, 184-185.

[4] Chiti F, Dobson CM (2017) Protein misfolding, amyloid formation, and human disease: A summary of progress over the last decade. Annu Rev Biochem 86, 27-68.

[5] Minton AP (2000) Implications of macromolecular crowding for protein assembly. Curr Opin Struct Biol 10, 34-39.

[6] Yeung PS, Axelsen PH (2012) The crowded environment of a reverse micelle induces the formation of $\beta$-strand seed structures for nucleating amyloid fibril formation. $J \mathrm{Am}$ Chem Soc 134, 6061-6063.

[7] Knowles TP, White DA, Abate AR, Agresti JJ, Cohen SI, Sperling RA, De Genst EJ, Dobson CM, Weitz DA (2011) Observation of spatial propagation of amyloid assembly from single nuclei. Proc Natl Acad Sci U S A 108, 1474614751.

[8] Langer F, Eisele YS, Fritschi SK, Staufenbiel M, Walker LC, Jucker M (2011) Soluble A $\beta$ seeds are potent inducers of cerebral $\beta$-amyloid deposition. J Neurosci 31, 1448814495.

[9] Jucker M, Walker LC (2013) Self-propagation of pathogenic protein aggregates in neurodegenerative diseases. Nature 501, 45-51.

[10] Walker LC (2016) Proteopathic strains and the heterogeneity of neurodegenerative diseases. Anпи Rev Genet 50, 329-346.

[11] Prusiner SB (1991) Molecular biology of prion diseases. Science 252, 1515-1522.

[12] Colby D, Prusiner S (2011) De novo generation of prion strains. Nat Rev Microbiol 9, 771-777.

[13] Liu P, Reed MN, Kotilinek LA, Grant MK, Forster CL, Qiang W, Shapiro SL, Reichl JH, Chiang AC, Jankowsky JL, Wilmot CM, Cleary JP, Zahs JR, Ashe KH (2015) Quaternary structure defines a large class of amyloid$\beta$ oligomers neutralized by sequestration. Cell Rep 11, 1760-1771.

[14] Hayden EY, Teplow DB (2013) Amyloid $\beta$-protein and Alzheimer's disease. Alzheimers Res Ther 5, 60.

[15] Nasica-Labouze J, Nguyen PH, Sterpone F, Berthoumieu O, Buchete NV, Coté S, De Simone A, Doig AJ, Faller P, Garcia A, Laio A, Li MS, Melchionna S, Mousseau N, Mu Y, Paravastu A, Pasquali S, Rosenman DJ, Strodel B, Tarus B, Viles JH, Zhang T, Wang C, Derreumaux P (2015) Amyloid $\beta$ protein and Alzheimer's disease: When computer simulations complement experimental studies. Chem Rev 115, 3518-3563.

[16] Cheon M, Chang I, Mohanty S, Luheshi LM, Dobson CM, Vendruscolo M, Favrin G (2007) Structural reorganisation and potential toxicity of oligomeric species formed during the assembly of amyloid fibrils. PLoS Comput Biol 3, 17271738.

[17] Fu Z, Aucoin D, Davis J, Van Nostrand WE, Smith, SO (2015) Mechanism of nucleated conformational conversion of A 342 Biochemistry 54, 4197-4207.

[18] Knowles TP, Vendruscolo M, Dobson CM (2014) The amyloid state and its association with protein misfolding disease. Nat Rev Mol Cell Biol 15, 384-396.

[19] Young LM, Ashcroft AE, Radford SE (2017) Small molecule probes of protein aggregation. Curr Opin Chem Biol 39, 90-99.

[20] Rodriguez RA, Chen LY, Plascencia-Villa G, Perry G (2017) Elongation affinity, activation barrier, and stability of $A \beta 42$ oligomers/fibrils in physiological saline. Biochem Biophys Res Commun 487, 444-449.

[21] Arosio P, Knowles TP, Linse S (2015) On the lag phase in amyloid fibril formation. Phys Chem Chem Phys 17, 76067618.

[22] Dunstan DE, Hamilton-Brown P, Asimakis P, Ducker W, Bertolini J (2009) Shear flow promotes amyloid-beta fibrilization. Protein Eng Des Sel 22, 741-746.

[23] Trumbore, CN (2016) Shear-induced amyloid formation in the brain: I. Potential vascular and parenchymal processes. $J$ Alzheimers Dis 54, 457-470.

[24] Wolak DJ, Thorne RG (2013) Diffusion of macromolecules in the brain: Implications for drug delivery. Mol Pharm 10, 1492-1504.

[25] Ashton L, Dusting J, Imomoh E, Balabani S, Blanch EW (2010) Susceptibility of different proteins to flowinduced conformational changes monitored with Raman spectroscopy. Biophys J 98, 707-714.

[26] Luheshi LM, Dobson CM (2009) Bridging the gap: From protein misfolding to protein misfolding diseases. FEBS Lett 583, 2581-2586. 
[27] Dobson CM (2017) The amyloid phenomenon and its links with human disease. Cold Spring Harb Perspect Biol 9, a023648.

[28] Iliff JJ, Wang M, Liao Y, Plogg BA, Peng W, Gundersen GA, Benveniste H, Vates GE, Deane R, Goldman SA, Nagelhus EA, Nedergaard M (2012) A paravascular pathway facilitates CSF flow through the brain parenchyma and the clearance of interstitial solutes, including amyloid $\beta$. Sci Transl Med 4, 147ra111.

[29] Hladky SB, Barrand MA (2014) Mechanism of fluid movement into, through and out of the brain: Evaluation of the evidence. Fluids Barriers CNS 11, 26.

[30] Brinker T, Stopa E, Morrison J, Klinge P (2014) A new look at cerebrospinal fluid circulation. Fluids Barriers CNS 11, 10.

[31] Xie L, Kang H, Kang H, Xu Q, Chen MJ, Liao Y, Thiyagarajan M, O'Donnell J, Christensen DJ, Nicholson C, Iliff JJ, Takano T, Deane R, Nedergaard M (2013) Sleep drives metabolite clearance from the adult brain. Science 342, 373377.

[32] Gospodarczyk W, Kozak M (2017) Microchip circulation drastically accelerates amyloid aggregation of 1-42 $\beta$-amyloid peptide from Felis catus. ACS Chem Neurosci 8, 2558-2567.

[33] Dobson J, Kumar A, Willis LF, Tuma R, Higazi DR, Turner R, Lowe DC, Ashcroft AE, Radford SE, Kapura N, David J, Brockwell DJ (2017) Inducing protein aggregation by extensional flow. Proc Natl Acad Sci U S A 114, 4673-4678.

[34] Uversky VN (2017) Intrinsic disorder here, there, and everywhere, and nowhere to escape from it. Cell Mol Life Sci 74, 3065-3067.

[35] Dobson CM (2003) Protein folding and misfolding. Nature 426, 884-890.

[36] Raskatov JA, Teplow DB (2017) Using chirality to probe the conformational dynamics and assembly of intrinsically disorder amyloid proteins. Sci Rep 7, 12433.

[37] Menon S, Sengupta N (2017) Computational approaches to understanding the biological behaviour of intrinsically disordered proteins. Curr Sci 112, 1444-1454.

[38] Trumbore CN (2017) Shear-induced amyloid formation in the brain: II. An experimental system for monitoring amyloid shear processes and investigating potential spinal tap problems. J Alzheimers Dis 59, 543-557.

[39] Bekard IB, Asimakis P, Bertolini J, Dunstan DE (2011) The effects of shear flow on protein structure and function. Biopolymers 95, 733-745.

[40] Cohen SI, Linse S, Luheshi LM, Hellstrand E, White A, Rajah L, Otzen DE, Vendruscolo M, Dobson CM, Knowles TP (2013) Proliferation of amyloid- $\beta 42$ aggregates occurs through a secondary nucleation mechanism. Proc Natl Acad Sci U S A 110, 9758-9763.

[41] Qiang W, Kelley K, Tycko R (2013) Polymorph-specific kinetics and thermodynamics of $\beta$-amyloid fibril growth. $J$ Am Chem Soc 135, 6860-6871.

[42] Ferrone FA (2015) Assembly of $A \beta$ proceeds via monomeric nuclei. J Mol Biol 427, 287-290.

[43] Smith DE, Babcock HP, Chu S (1999) Single-polymer dynamics in steady shear flow. Science 283, 1724-1727.

[44] Lv Z, Roychaudhuri R, Condron MM, Teplow DB, Lyubchenko YL (2013) Mechanism of amyloid $\beta$-protein dimerization determined using single-molecule AFM force spectroscopy. Sci Rep 3, 2880.

[45] Iyengar SS, Sumner I, Jakowski J (2008) Hydrogen tunneling in an enzyme active site: A quantum wavepacket dynamical perspective. J Phys Chem B 112, 7601-7613.
[46] Nedergaard M (2013) Neuroscience. Garbage truck of the brain. Science 340, 1529-1530.

[47] Iliff JJ, Lee H, Yu M, Feng T, Logan J, Nedergaard M, Benveniste H (2013) Brain-wide pathway for waste clearance captured by contrast-enhanced MRI. J Clin Invest 123, 1299-1309.

[48] Trumbore CN, Tremblay RD, Penrose JT, Mercer M, Kelleher FM (1983) Unusual flow behavior in high-performance liquid chromatography capillary tubing. J Chromatogr $\mathbf{2 8 0}$, 43-57.

[49] Lesne SE (2014) Toxic oligomer species of amyloid- $\beta$ in Alzheimer's disease, a timing issue. Swiss Med Wkly 144, w14021.

[50] Dreha-Kulaczewski S, Joseph AA, Merboldt KD, Ludwig HC, Gartner J, Frahm J (2015) Inspiration is the major regulator of human CSF flow. J Neurosci 35, 2485-2491.

[51] Faubel R, Westendorf C, Bodenschatz E, Eichele1 G (2016) Cilia-based flow network in the brain ventricles. Science 353, 176-178.

[52] Stone J, Johnstone DM, Mitrofanis J, O'Rourke M (2015) The mechanical cause of age-related dementia (Alzheimer's disease): The brain is destroyed by the pulse. J Alzheimers Dis 44, 355-373.

[53] Mannini B, Chiti F (2017) Chaperones as suppressors of protein misfolded oligomer toxicity. Front Mol Neurosci 10, 98.

[54] Dubnikov T, Ben-Gedalya T, Cohen E (2017) Protein quality control in health and disease. Cold Spring Harb Perspect Biol 9, a023523.

[55] Spires-Jones TL, Attems J, Thal DR (2017) Interactions of pathological proteins in neurodegenerative diseases. Acta Neuropathol 134, 187-205.

[56] Sepulcher J, Schultz AP, Sabuncu M, Gomez-Isla T, Chhatwal J, Becker A, Sperling R, Johnson KA (2016) In vivo tau, amyloid, and gray matter profiles in the aging brain. $J$ Neurosci 36, 7364-7374.

[57] Pickett EK, Koffie RM, Wegmann S, Henstridge CM, Herrmann AG, Colom-Cadena M, Lleo A, Kay KR, Vaught M, Soberman R, Walsh DM, Hyman BT, Spires-Jones TL (2016) Non-fibrillar oligomeric amyloid- $\beta$ within synapses $J$ Alzheimers Dis 53, 787-800.

[58] Yang M, Teplow DB (2008) Amyloid beta-protein monomer folding: Free-energy surfaces reveal alloform-specific differences. J Mol Biol 384, 450-464.

[59] Tsien RY (2013) Very long-term memories may be stored in the pattern of holes in the perineuronal net. Proc Natl Acad Sci U S A 110, 12456-12461.

[60] Tirrell M, Middleman S (1975) Shear modification of enzyme kinetics. Biotechnol Bioeng 17, 299-303.

[61] Goldmann WH, Guttenberg Z, Ezzell RM, Isenberg G (1998) The study of fast reactions by the stopped flow method. In Modern Optics, Electronics and High Precision Techniques in Cell Biology. Isenberg G, ed. Springer, Berlin, Heidelberg, pp. 159-171.

[62] Bernstein SL, Dupuis NF, Lazo ND, Wyttenbach T, Condron MM, Bitan G, Teplow DB, Shea JE, Ruotolo BT, Robinson CV, Bowers MT (2009) Amyloid- $\beta$ protein oligomerization and the importance of tetramers and dodecamers in the aetiology of Alzheimer's disease. Nat Chem 1, 326-331.

[63] Metzner AB, Cohen Y, Rangel-Nafaile C (1979) Inhomogeneous flows of non-Newtonian fluids: Generation of spatial concentration gradients. J Nonnewton Fluid Mech 5, 449462 . 